\title{
Gegen Qinlian Decoction Alleviates Experimental Colitis and Concurrent Lung Inflammation by Inhibiting the Recruitment of Inflammatory Myeloid Cells and Restoring Microbial Balance
}

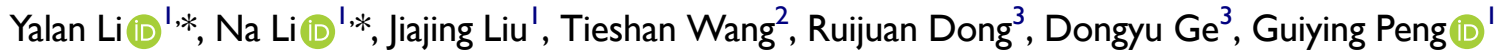 \\ 'Department of Immunology and Microbiology, School of Life Sciences, Beijing University of Chinese Medicine, Beijing, People's Republic of China; \\ ${ }^{2}$ Beijing Research Institute of Chinese Medicine, Beijing University of Chinese Medicine, Beijing, People's Republic of China; ${ }^{3}$ Experimental Teaching \\ Center, School of Traditional Chinese Medicine, Beijing University of Chinese Medicine, Beijing, People's Republic of China
}

*These authors contributed equally to this work

Correspondence: Guiying Peng, Email penggy@bucm.edu.cn

\begin{abstract}
Objective: Ulcerative colitis (UC) as one of the intractable diseases in gastroenterology seriously threatens human health. Respiratory pathology is a representative extraintestinal manifestation of UC affecting the quality of life of patients. Gegen Qinlian Decoction (GQD) is a classical traditional Chinese medicine prescription for UC or acute lung injury. This study was aimed to reveal the therapeutic effect of GQD on UC and its pulmonary complications and uncover its molecular mechanism mediated by myeloid cells and microbiota.

Methods: Mice with DSS-induced colitis were orally administrated with GQD. Overall vital signs were assessed by body weight loss and disease activity index (DAI). Pulmonary general signs were evaluated by pulmonary pathology and lung function. The mechanism of GQD relieving UC was characterized by detecting myeloid cells (neutrophils, macrophages, inflammatory monocytes, and resident monocytes) in colonic and lung tissues, related inflammatory cytokines, as well as the microbiota in bronchoalveolar lavage fluid (BALF) and feces.

Results: GQD significantly reduced weight loss, DAI scores, and lung injury but improved the lung function of colitis mice. The DSS-induced colonic and concurrent pulmonary inflammation were also alleviated by GQD, as indicated by the down-regulated expressions of inflammatory cytokines (TNF- $\alpha$, IL-1 $\beta$, IL-6, CCR2, and CCL2) and the suppressed recruitment of neutrophils and inflammatory monocytes. Meanwhile, GQD greatly improved intestinal microbiota imbalance by enriching Ruminococcaceae UCG013 while decreasing Parabacteroides, [Eubacterium]_fissicatena_group, and Akkermansia in the feces of colitis mice. Expectantly, GQD also restored lung microbiota imbalance by clearing excessive Coprococcus 2 and Ochrobactrum in the BALF of colitis mice. Finally, significant correlations appeared between GQD-mediated specific bacteria and inflammatory cytokines or immune cells.

Conclusion: GQD could alleviate UC by decreasing excessive inflammatory myeloid cells and cytokines, and reshaping the microbiota between the colon and lung, which contributes to clarifying the mechanism by which GQD ameliorates colitis-associated pulmonary inflammation.
\end{abstract}

Keywords: Gegen Qinlian Decoction, ulcerative colitis, lung and intestinal microbiota, pulmonary inflammation, myeloid cells

\section{Introduction}

Ulcerative colitis (UC), the most common form of inflammatory bowel disease (IBD), is a chronic nonspecific inflammation of the colon and rectum, with an increasing incidence worldwide. ${ }^{1}$ The clinical manifestations of IBD include intestinal and extra-intestinal manifestations, with extra-intestinal manifestations occurring in up to $40 \%$ of IBD patients. ${ }^{2}$ Numerous researchers have found that IBD-related lung injury cannot be ignored, ${ }^{3,4}$ and UC was more susceptible to respiratory complications than Crohn's disease (CD). ${ }^{5}$ According to statistics, up to $37 \%$ to $55 \%$ of IBD patients have abnormalities on lung function tests or lung imaging. ${ }^{6,7}$ Furthermore, Kraft et al described the pulmonary manifestations of IBD with multiple 
different phenotypes, such as airway disease, pulmonary parenchymal disease, and pulmonary vascular disease. ${ }^{8}$ Thus, the reduction of pulmonary symptoms is essential to enhance the life quality of IBD patients.

In the last decades, growing studies have suggested that the "gut-lung axis" plays an important role in IBD-derived lung injury. ${ }^{9,10}$ Cytokine levels and epithelial cell destruction in the intestinal epithelium after exposure to airway antigens presented consistent changes with those in the lung epithelium. This may be the cause of lung injury during the progress of IBD because of the similar inflammatory responses between them. ${ }^{11}$ In addition, abnormal migration of inflammatory cells to other organs might explain the extraintestinal appearance of IBD. ${ }^{12,13}$

Myeloid cells generated by bone marrow hematopoietic stem cells, including neutrophils, monocytes, and macrophages, are considered as effector cells of inflammation and serve as governors in the immune regulation of IBD. ${ }^{14}$ During intestinal inflammation, neutrophils are first activated and recruited to the site of infection, which further damages the integrity of the epithelial barrier. ${ }^{15-17}$ Subsequently, inflammatory monocytes are recruited to the inflamed site, differentiate into inflammatory monocytes/macrophages, and release pro-inflammatory cytokines (such as TNF- $\alpha$, IL-1 $1 \beta$, and IL-6, etc.). ${ }^{17-19}$ This recruitment process would prolong the inflammatory response which could be regulated by the CCL2-CCR2 axis. ${ }^{20}$ Therefore, the inhibition of the recruitment of inflammatory myeloid cells may be a key approach for the treatment of colitis.

On the other hand, the gut microbiota is widely known to be closely related to intestinal health. Compared with healthy controls, lower microbial diversity, fewer predominant Firmicutes, and enriched pro-inflammatory Proteobacteria have been observed in the intestine of IBD patients. ${ }^{21}$ Pulmonary microbes such as Gammaproteobacteria are also critical during the progress of respiratory diseases, using their inflammatory byproducts to survive and thrive in anaerobic and hypoxic conditions. ${ }^{22,23}$ Based on these findings, we hypothesized that this interaction between resident microbiota and immune responses might be further involved in regulating the occurrence of lung injury in colitis.

Gegen Qinlian Decoction (GQD), a classical traditional Chinese medicine (TCM) formula, has been clinically proven to be effective in the treatment of UC. A meta-analysis involving 2028 patients with UC showed that GQD alone or combined with western medicine was significantly superior to western medicine alone in treating UC, with low recurrence rates and adverse events. ${ }^{24}$ In vivo and in vitro experiments have shown that GQD exerts anti-inflammatory, antioxidant stress, antibacterial, antidiarrheal, regulating gastrointestinal function, and other pharmacological effects. ${ }^{25}$ Several active components of GQD, such as baicalin, glabridin, and berberine, contributed to exerting these immunomodulatory effects. ${ }^{25-28}$ Network pharmacological studies have also revealed that GQD could alleviate the UC-related inflammation via inhibiting the production of proinflammatory cytokines including IL-1 $\beta$, TNF-a, and IL- $6 .{ }^{29}$ In addition, GQD effectively improved pulmonary edema and microvascular permeability as well as inhibited lung inflammation and apoptosis in mice with LPS-induced acute lung injury. ${ }^{30}$ Nonetheless, the underlying molecular mechanism by which GQD alleviates lung inflammation associated with colitis remains unclear.

In the present study, we for the first time investigated the therapeutic effect of GQD on pulmonary injuries in addition to the colon in UC mice. The mechanisms by which GQD maintains the immune homeostasis mediated by myeloid immune cells and resident microbiota were explored. The findings of this research will facilitate the discovery of new therapeutic biomarkers and adjuvant medications for clinical colitis with lung complications.

\section{Materials and Methods \\ Preparation of GQD}

We selected the Gegen Qinlian dispensing granule as the medication for experimental mice, a pharmaceutical form of Gegen Qinlian Decoction (GQD), which is effective in treating UC in our previous studies. ${ }^{31}$ Gegen Qinlian dispensing granule was purchased from China Resources Sanjiu Medical \& Pharmaceutical Co., Ltd. The human dose of formula granule was $0.12 \mathrm{~g} / \mathrm{kg}$ and was diluted with distilled water (DW).

\section{Animals and Treatment}

A total of 32 female C57BL/6J mice (20-22 g), purchased SPF Biotechnology Co., Ltd. (Beijing, China), were randomly divided into 4 groups including control, DSS, DSS+GQD, and DSS+sulfasalazine (SASP) with 8 mice per group. Except 

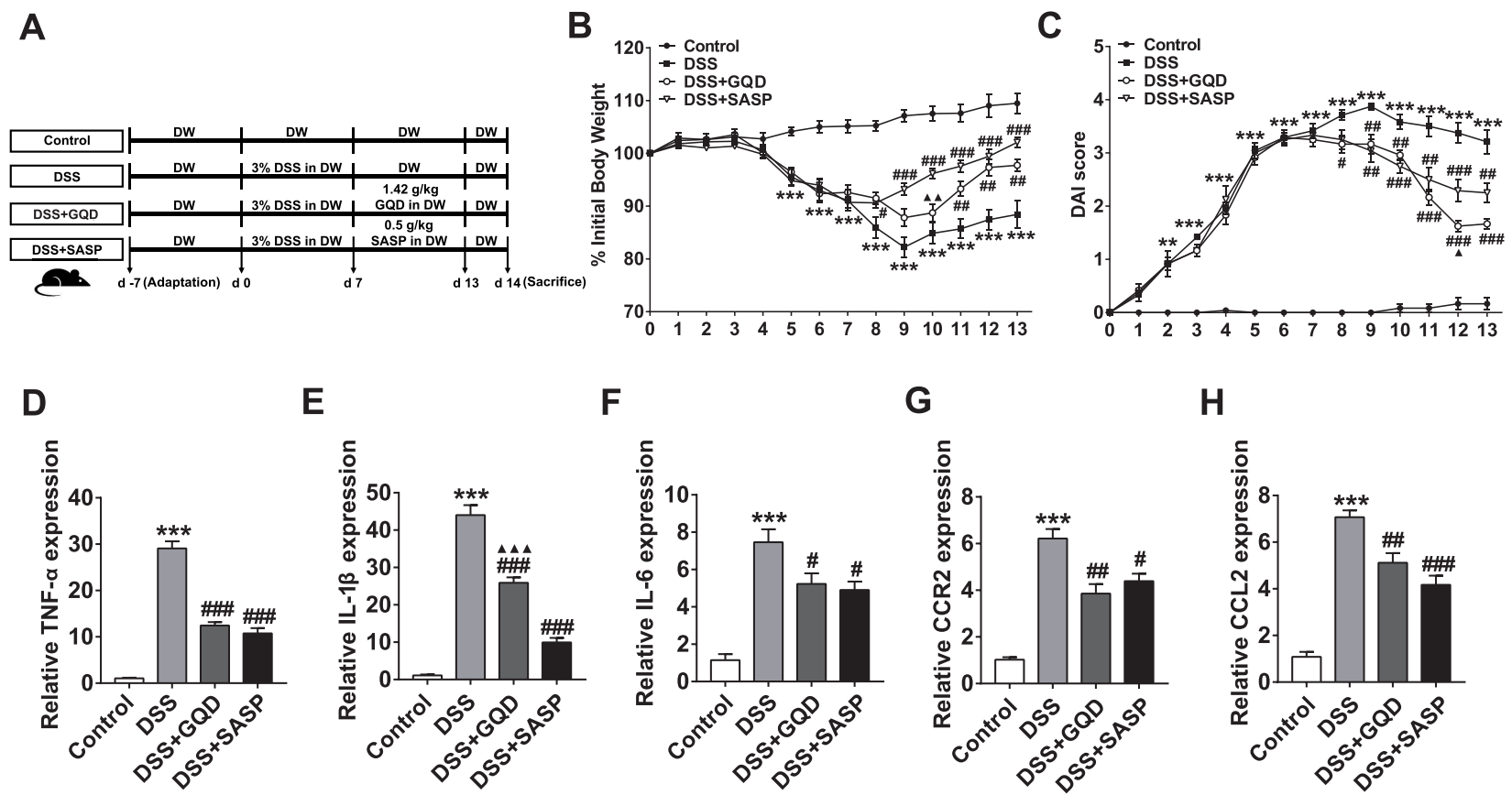

Figure I GQD alleviates the colonic inflammation of DSS-induced colitis mice. (A) The time course of DSS administration and different treatment in mice. C57BL/6J mice were provided with water or 3\% DSS-containing water from days 0 to 6 . On days 7 to 13, mice were orally administered with GQD or SASP. (B) Body weight changes in each group. (C) DAl scores in each group. The mRNA relative expressions of inflammatory cytokines including TNF- $\alpha(\mathbf{D})$, IL-I $\beta$ (E), IL-6 (F), CCR2 (G), and CCL2 (H) in colonic tissues were assessed by RT-qPCR. Data were expressed as mean \pm SEM $(n=4-8)$. ${ }^{* * P}<0.0 \mathrm{I}$, ${ }^{* * *} P<0.00 \mathrm{I}$, compared with the control group; ${ }^{\#} P<0.05$, ${ }^{\#} P<0.0 \mathrm{I}$, and $P<0.00$ I, compared with the DSS group; ${ }^{\Delta} P<0.05, \Delta{ }^{\Delta} P<0.01, \Delta \Delta \Delta_{P}<0.00$ I, compared with the SASP group.

for the control group, experimental colitis mice in the other three groups were induced by the administration with $3 \%$ (w/ v) DSS (MP Biomedicals, MW: 36,000-50,000) ad libitum in drinking water for consecutive 7 days. On days 7 to 13 , mice in the DSS+GQD group were orally administered with $1.42 \mathrm{~g} / \mathrm{kg}$ of formula granules according to the clinical equivalent dose and given $200 \mu \mathrm{L}$ per mouse. The DSS+SASP group was orally given $0.5 \mathrm{~g} / \mathrm{kg}$ of SASP. Mice in the control and DSS groups were administered with distilled water. The changes in body weight and fecal traits were recorded daily. At the end of the experiment, mice were euthanized by intraperitoneal injection with $0.25 \mathrm{~g} / \mathrm{kg}$ sodium pentobarbital, ${ }^{32}$ and the colon and lung tissues were collected for further analysis. The timeline of this test is shown in Figure 1A. All procedures involving animals were approved by the Animal Ethics Committee of Beijing University of Chinese Medicine (BUCM-4-2019090303-3097), following guidelines issued by Regulations of Beijing Laboratory Animal Management.

\section{Evaluation of Disease Activity Index}

The disease activity index (DAI) was determined daily by body weight loss, stool consistency, and the detection of rectal bleeding according to a standard scoring system. ${ }^{33}$ The DAI sore is equivalent to the average of the above three indicators.

\section{Histopathological Evaluation}

Lungs were washed in phosphate buffer, fixed in $10 \%$ formaldehyde at room temperature, dehydrated in a graded concentration of ethanol, and then embedded in paraffin. Tissue sections of $4 \mu \mathrm{m}$ were stained with hematoxylin and eosin (H\&E) to evaluate the histopathological changes of the lung. Digital images of pulmonary morphology at $200 \times$ magnification were obtained using a light microscope and graded according to the reference. ${ }^{34}$

\section{Assessment of Pulmonary Function}

Respiratory function was measured using the flexiVent (SCIREQ, Montreal, QC, Canada) and all operations were performed according to the manufacturer's instructions. Mice were anesthetized by $0.1 \mathrm{~g} / \mathrm{kg}$ pentobarbital sodium to 
prevent spontaneous respirations and connected to a computer-controlled animal ventilator through a tracheal cannula. The following measures of respiratory mechanics were calculated: Respiratory system resistance (Rrs), elasticity resistance (Ers), compliance (Crs), the resistance of the central airways (Rn), tissue damping $(\mathrm{G})$, tissue elastance $(\mathrm{H})$, and static compliance (Cst). Data were recorded continuously and analyzed offline. The airway physiology testing procedure took approximately $10 \mathrm{~min}$ per mouse, with an approximate surgery time of $5 \mathrm{~min}$ per animal.

\section{Cell Isolation from Lung and Intestinal Tissues in Mice}

For the isolation of myeloid cells from the lung and colon, the single-cell suspension was obtained according to a previous study. ${ }^{35}$ Fresh lung tissues were minced and incubated with $1 \mathrm{mg} / \mathrm{mL}$ collagenase IV (Worthington, USA) and $50 \mu \mathrm{g} / \mathrm{mL}$ DNase I (Roche, Switzerland) in RPMI-1640 media (Biological Industries, Israel) before being mashed through $70 \mu \mathrm{m}$ cell strainers. After removing adherent fat tissue and Peyer's patches, the colon was washed twice with $20 \mathrm{~mL}$ HBSS medium containing $5 \mathrm{mM}$ EDTA and $1 \mathrm{mM}$ DTT to remove epithelial cells. Next, $2 \mathrm{mg} / \mathrm{mL}$ collagenase type III (Worthington, USA) and $50 \mu \mathrm{g} / \mathrm{mL}$ DNase I (Roche, Switzerland) in RPMI-1640 media were used to digest colon tissues. The digested tissues were filtered through $70 \mu \mathrm{m}$ cell strainers to obtain cell suspension and enriched with a 40\% Percoll gradient after red blood cells were lysed. Single-cell suspensions from all tissues were used for subsequent flow cytometry staining.

\section{Flow Cytometry Analysis}

All staining of molecules with fluorescently labeled antibodies was performed in the dark. All antibodies were purchased from Biolegend Ltd., U. S. A. Single-cell suspensions were kept at $4{ }^{\circ} \mathrm{C}$, and nonspecific binding was blocked by anti-Fc receptor blocking antibody (anti-CD16/32) before cell surface was stained with fluorescent conjugated antibodies. Then, cells were stained with PB anti-mouse CD45, PE-Cy5 anti-mouse CD11b, APC-Cy7 anti-mouse Ly6C, FITC anti-mouse CD11c, APC anti-mouse Ly6G, F4/80 anti-mouse AF700. Cells were detected by FACSCantoTM (BD Biosciences, U.S. A) and analyzed by FlowJo software.

\section{RNA Isolation, cDNA Synthesis, and Real-Time Quantitative PCR}

Total RNA from lung and colonic tissues was extracted using RNAsimple Total RNA Kit (TIANGEN, China). RNA was quantified by a Nanodrop ND-1000 Spectrophotometer (Thermo Fisher Scientific, U.S.A) and then reversely transcribed into cDNA using QuantiNova Reverse Transcription Kit (Qiagen Ltd., Germany). RT-qPCR was performed in a QuantStudio6 Flex system (Life Technologies, U.S.A) with QuantiNova SYBR Green PCR Kit (Qiagen Ltd., Germany). The mRNA expressions of targeted genes were normalized using a housekeeping control (GAPDH) and calculated by the $2^{-\Delta \Delta \mathrm{Ct}}$ method. Specific primers used in this study were as follows: 5'-AGACACCATGAGCACAGAAAGC-3'/5'-CCATA GAACTGATGAGAGGGAGG-3' for TNF- $\alpha$; 5'-ACAAGAGCTTCAGGCAGGCAGTATC-3'/5'-TATGGGTCC GACAGCACGAGGC-3' for IL-1 $\beta$; 5'-TGCAATGGCAATTCTGATTGTATG-3'/5'-TGTATCTCTCTGAAGGACTCTG3' for IL-6; 5'-TTTGTTTTTGCAGATGATTCAA-3'/5'-TGCCATCATAAAGGAGCCAT-3' for CCR2. 5'-CCCAA TGAGTAGGCTGGAGA-3'/5'-TCTGGACCCATTCCTTCTTG-3' for CCL2; 5'-GCACCACCAACTGCTTAG-3'/5'GGATGCAGGGATGATGTTC-3' for GAPDH.

\section{DNA Extraction, I6S rRNA Gene Sequencing, and Data Analysis}

Fresh feces were sampled from all the mice. Then, fresh bronchoalveolar fluid (BALF) was collected after mice were euthanized. Total genomic DNA was isolated from BALF and feces using the QIAamp DNA Stool Mini Kit (Qiagen Ltd., Germany) according to the manufacturer's instructions. The V3-V4 regions of the 16S rRNA gene were amplified using universal primers 343F (TACGGRAGGCAGCAG) and 798R (AGGGTATCTAATCCT). After purification and quantification, the PCR products were pooled and adjusted into equimolar amounts and sequenced on an Illumina NovaSeq6000 sequencer to produce paired-end reads of $250 \mathrm{bp}$.

Initial reads were de-multiplexed and quality-filtered using the QIIME platform (version 1.9). Briefly, low-quality sequences with a length of $<220 \mathrm{nt}$ or $>500 \mathrm{nt}$, an average quality score of $<20$, and sequences containing $>3$ nitrogenous bases were abandoned. After removing chimera using UCHIME, the remaining clean reads were then 
clustered into operational taxonomic units (OTUs) using VSEARCH (version 7.1) with a 97\% similarity cutoff. Taxonomy assignment of OTUs was performed against the SILVA 16S rRNA gene database (Release 132) at a confidence threshold of $70 \%$.

Sobs and Shannon indexes were calculated by QIIME (version 1.9) to estimate alpha diversity. Bar plots were visualized to present the bacterial community composition using the "vegan" package in R (version 3.3.1). Principal coordinates analysis (PCoA) was performed based on Bray-Curtis and Jaccard distances using QIIME (version 1.9) to evaluate beta diversities. In addition, permutational multivariate analysis of variance (PERMANOVA, with 1000 Monte Carlo permutations) was carried out based on distance matrices to compare the difference of community structures between groups using the Adonis function available in the "vegan" package of R (version 3.3.1). The differentially abundant bacteria taxa among groups were identified using discriminant analysis (LDA) effect size (LEfSe) analysis. Only taxa with an average relative abundance greater than $0.01 \%$ were included. The microbial gene functions were predicted using PICRUSt (https://github.com/picrust). Differentially abundant KEGG pathways between groups were obtained using STAMP (version 2.1.3).

\section{Statistical Analysis}

Data were analyzed using SPSS (version 22.0, SPSS Inc., USA). Parametric data were analyzed using unpaired one-way ANOVA with Tukey's post hoc test. Nonparametric data were analyzed using the Kruskal-Wallis test. $P$ values for multiple comparisons were adjusted with a false discovery rate (FDR) according to Benjamini and Hochberg. ${ }^{39}$ Corrected $P$ values less than 0.05 were considered statistically significant. Data were presented as means and standard error of the mean (SEM). Spearman correlation coefficients between microbes and cytokines, microbes and immune cells, fecal microbes, and lung microbes, were estimated using the "psych" package in R (version 3.3.1). Significant correlations $(P<0.05)$ were visualized with network graphs using Gephi (version 0.9.2).

\section{Results}

\section{GQD Alleviates the Intestinal Inflammation of DSS-Induced Colitis Mice}

To determine the effect of GQD on colitis, DSS-induced colitis mice were orally given GQD, SASP, or distilled water intervention for 7 days (Figure 1A). Our results showed that colitis mice had significantly lower body weight but higher DAI scores compared with controls (Figure 1B and C, $P<0.01$ ). Expectantly, both GQD and SASP continuously restored the body weight and reduced the DAI scores of these colitis mice (Figure 1B and $\mathrm{C}, P<0.01$ ).

We next determined the mRNA expressions of the pro-inflammatory cytokines in the colon. Similarly, the relative expressions of these above cytokines and chemokines were significantly higher in the DSS group compared with control mice (Figure 1D-H, $P<0.001$ ). However, these alterations could be relieved by GQD and SASP (Figure 1D-H, $P<$ 0.05). Additionally, SASP decreased the production of IL-1 $\beta$ to a greater extent compared to GQD (Figure 1E, $P<$ $0.001)$.

\section{GQD Decreases Inflammatory Myeloid Cells in the Colon of DSS-Induced Colitis Mice}

We performed the flow cytometry analysis to the phenotype of myeloid cells in the colon of DSS-induced colitis mice. As shown in Figure 2, compared with the control, significant increases in the percentages of neutrophils, macrophages, and Ly6C $\mathrm{C}^{\text {hi }}$ inflammatory monocytes in the colon of DSS-induced colitis mice were observed (Figure 2A-D, $P<0.01$ ). The proportion of Ly6C $\mathrm{C}^{\mathrm{lo}}$ resident monocyte was not significantly altered (Figure $2 \mathrm{~A}$ and $\mathrm{E}, P>0.05$ ). As expected, the GQD and SASP treatments distinctly suppressed those augmentations equally in colitis mice (Figure $2 \mathrm{~A}-\mathrm{D}, P<0.05$ ).

\section{GQD Alleviates the Gut Microbiota Imbalance of DSS-Induced Colitis Mice}

We further examined the effects of GQD intervention on gut microbiota in DSS-induced colitis mice. At the overall community level, colitis mice with or without GQD intervention exhibited smaller Shannon and sobs indexes in the gut microbiota than controls (Figure 3A, $P<0.05$ ), indicating a lower community richness and evenness. PCoA plots further 
A
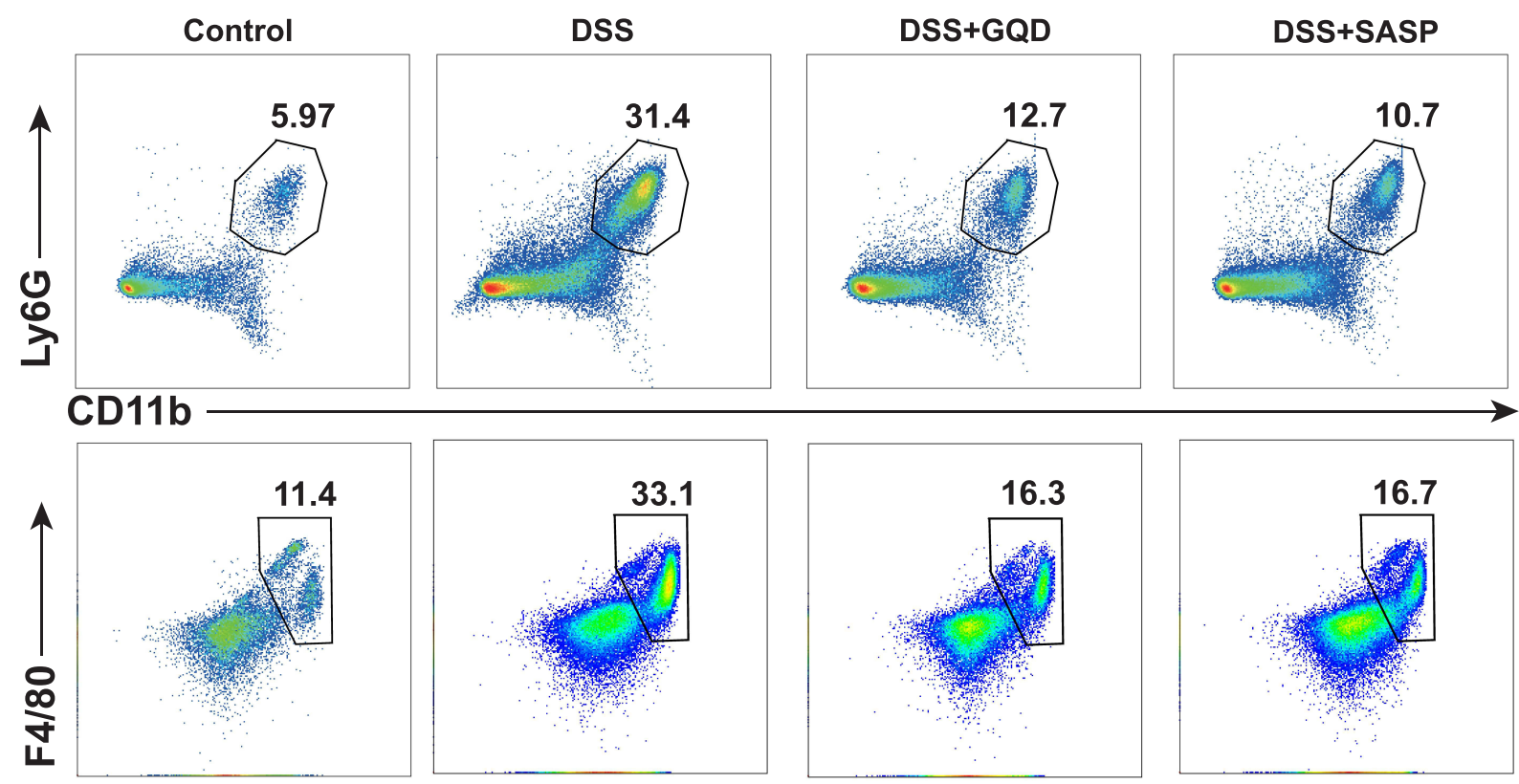

\section{CD11b}
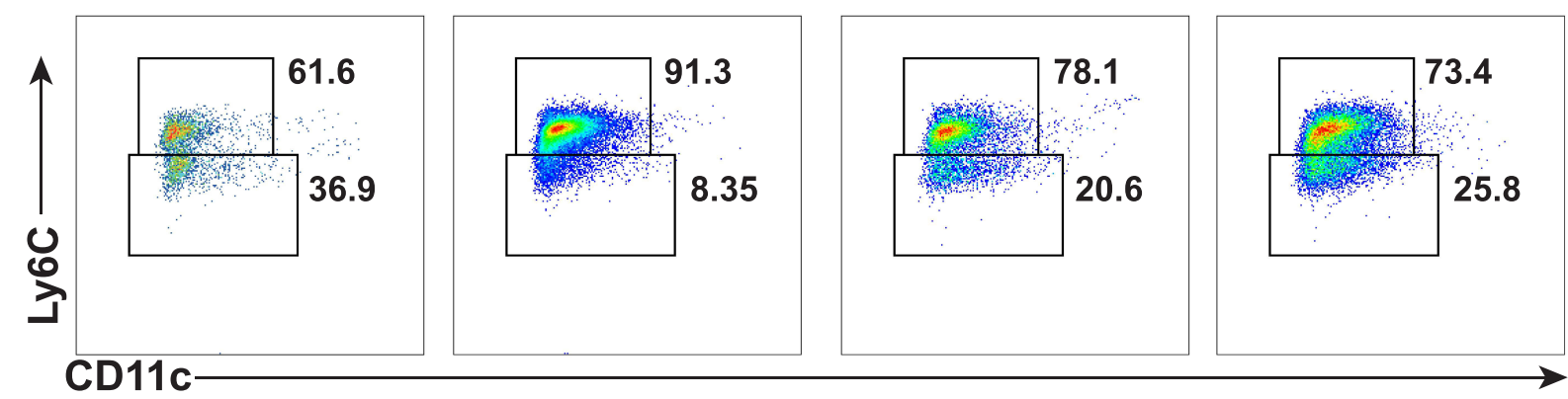

B

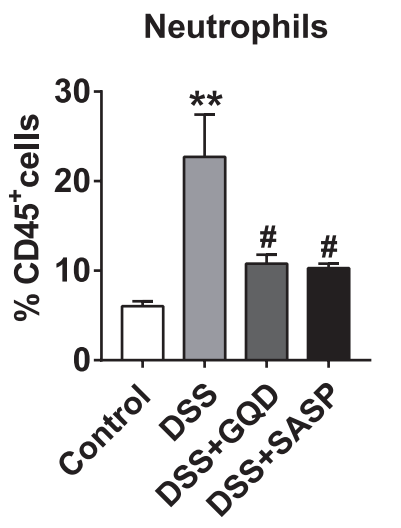

C

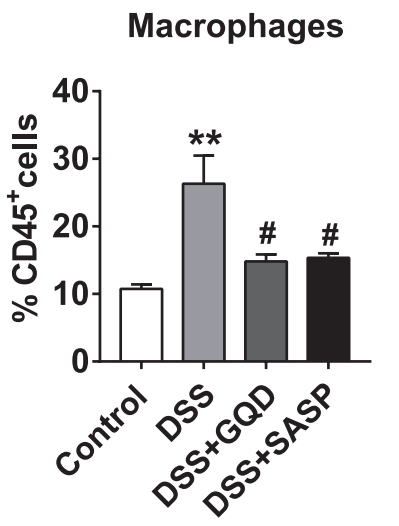

D Monocytes (Inflam)

E

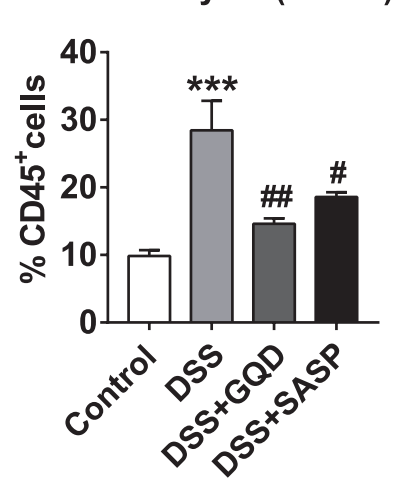

Monocytes (Res)

Figure 2 Myeloid cell populations in the colon among different groups. (A) Representative gating strategies for identifying myeloid cells in the colon quantified by flow cytometry. Neutrophils were identified as $\mathrm{CD}_{4} 5^{+} \mathrm{CDIIb}{ }^{+} \mathrm{Ly} 6 \mathrm{G}^{+}$cells, macrophages as $\mathrm{CD} 45^{+} \mathrm{CDII} \mathrm{b}^{+} \mathrm{F} 4 / 80^{+}$cells, inflammatory (Inflam) monocytes as

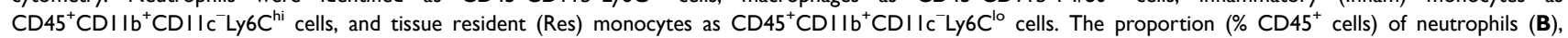
macrophages (C), inflammatory (Inflam) monocytes (D), and monocytes (Res) (E). Data were expressed as mean \pm SEM $(n=4)$. $* * P<0.01$, $* * * P<0.00 \mathrm{I}$, compared with the control group; ${ }^{\#} P<0.05,{ }^{\# \#} P<0.01$, compared with the DSS group. 
A
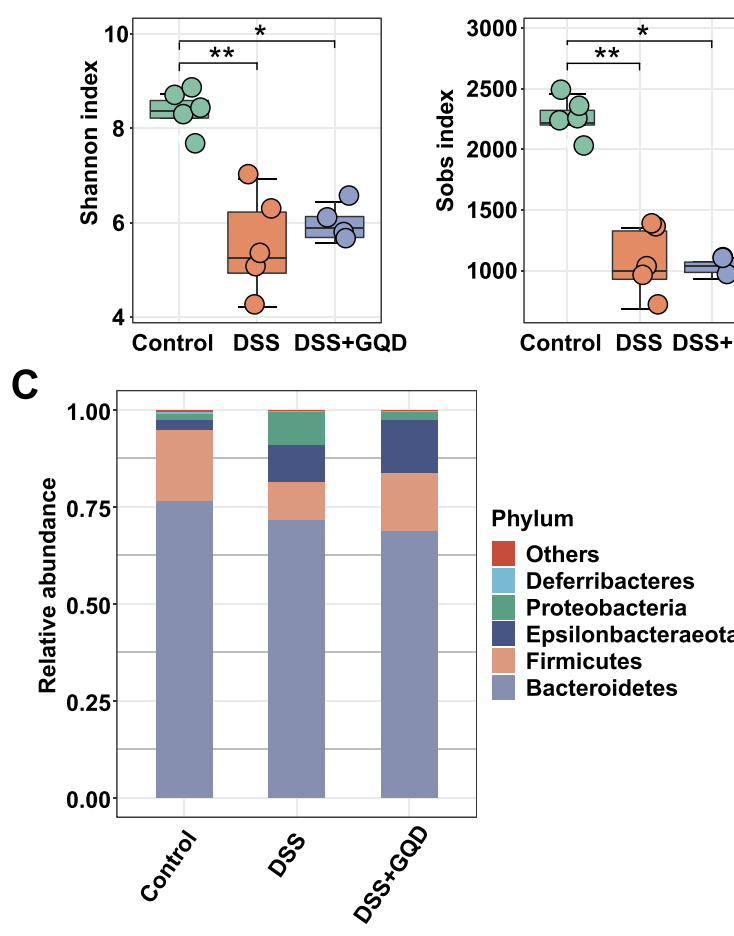

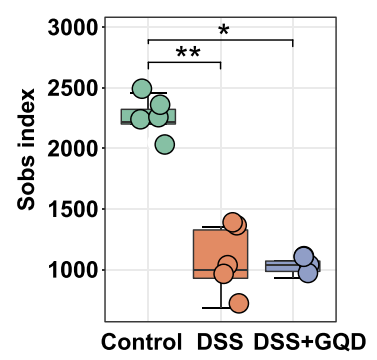

B

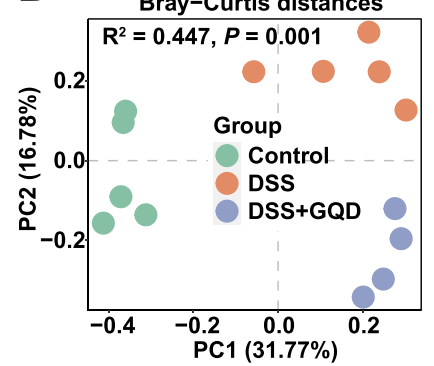

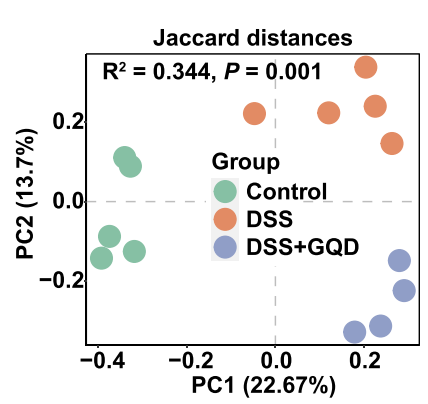

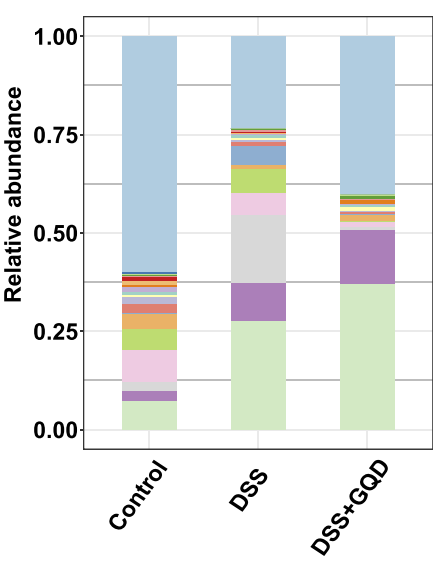

Genus

Others

Intestinimonas

Anaerotruncus

Roseburia

Prevotellaceae UCG-001

Lachnoclostridium

Ruminococcaceae UCG-014

Azospirillum sp._47_25

Parasutterella

Muribaculum

Esubacterium l coprosta

Shigella

Lachnospiraceae_NK4A136_group

Alistipes

Parabacteroides

Campylobacter

Bacteroides

Figure 3 The alterations in the colonic microbiota structure of colitis mice. (A) Shannon and sobs index. (B) Principal coordinate analysis (PCoA) plots based upon BrayCurtis distances and Jaccard distances. (C) Abundant phyla and genera $(n=4-5)$. $* P<0.05, * * P<0.01$, compared with the control group.

suggested the significant separation of fecal samples among three groups (Figure 3B). PERMONOVA based on these distances confirmed that the community structures were strongly affected by DSS and/or GQD supplementation (BrayCurtis distances, $\mathrm{R}^{2}=0.447, P=0.001$; Jaccard distances, $\left.\mathrm{R}^{2}=0.344, P=0.001\right)$. Moreover, a significant difference in the community composition was also seen among different groups (Figure $3 \mathrm{C}$ ).

Subsequently, we conducted the LEfSe analysis to search differentially abundant genera among three groups of mice. As shown in Figure 4A and B, the genus Ruminococcaceae_UCG-013, less abundant in colitis mice than controls, was significantly enriched by the GQD administration. On the contrary, Parabacteroides, [Eubacterium]_fissicatena_group, and Akkermansia exhibited higher relative proportions in the intestine of colitis mice compared to the control group. However, the GQD treatment remarkably suppressed the accumulation of these three microbes.

We subsequently predicted the gut microbial gene functions using PICRUST. We observed that the relative abundances of microbial genes associated with lysine biosynthesis as well as phenylalanine, tyrosine, and tryptophan biosynthesis were significantly down-regulated in colitis mice than controls (Figure 4C). However, the GQD addition reversed these alterations as reflected by the higher proportions of genes related to these two pathways in the DSS+GQD group.

The above outcomes demonstrated that the GQD administration might contribute to improving the gut microbiota imbalance to a certain extent via selectively regulating specific microbes and their underlying gene functions.

\section{GQD Alleviates Pulmonary Inflammation and Improves Lung Function in DSS-Induced Colitis Mice}

We further evaluated the pulmonary damage caused by DSS-induced colitis. As shown in Figure 5A, compared with controls, colitis mice exhibited the damaged alveolar structure, less common normal alveoli, fused and thickened alveolar walls, and expanded inflammatory cell infiltration around trachea bronchi and blood vessels. As well, the lung pathological scores were significantly higher in these colitis mice than controls (Figure 5B, $P<0.001$ ). However, the above adverse alterations were remarkably alleviated by the intervention of GQD (Figure 5B, $P<0.01$ ). Notably, the 
A

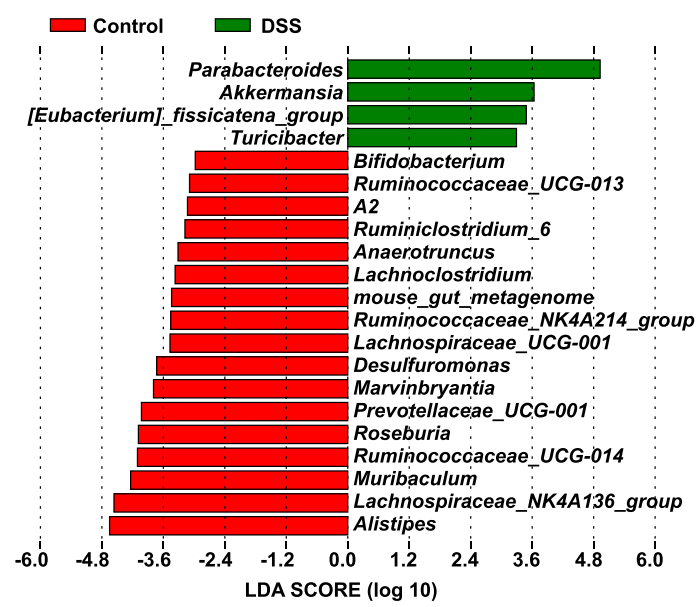

DSS DSS+GQD

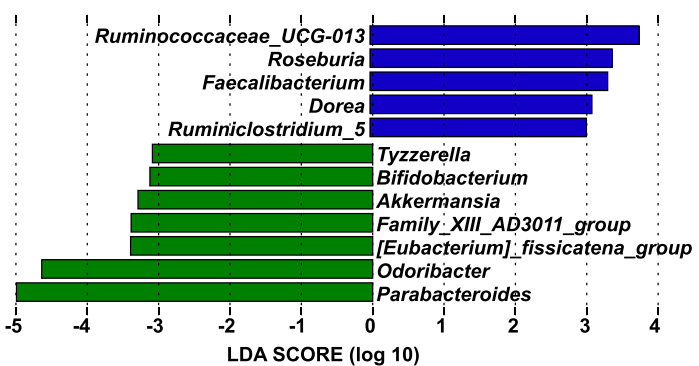

$\mathbf{B}_{\text {co }}$

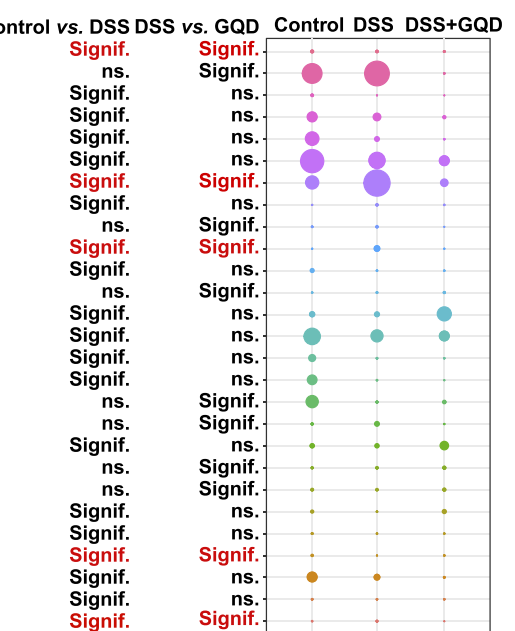

Genus

Bifidobacterium

Odoribacter

mouse_gut_metagenome

Muribaculum

Prevotellaceae_UCG-001

Alistipes

Parabacteroides

Turicibacter

Family_XIII_AD3011_group

[Eubacterium]_fissicatena_group

A2

Dorea

Lachnoclostridium

Lachnospiraceae NK4A136 grou

Lachnospiraceae_UCG-001

Marvinbryantia

Roseburia

Tyzzerella

Faecalibacterium

Ruminiclostridium 5

Ruminiclostridium 6

Ruminococcaceae_NK4A214_group Ruminococcaceae

Ruminococcaceae-UCG-014

Ruminococcaceae

Akkermansia

\begin{tabular}{|c|c|}
\hline $\begin{array}{l}\text { Family } \\
\text { Bifidobacteriaceae } \\
\text { Marinifilaceae } \\
\text { Muribaculaceae } \\
\text { Muribaculaceae } \\
\text { Prevotellaceae } \\
\text { Rikenellaceae } \\
\text { Tannerellaceae } \\
\text { Erysipelotrichaceae } \\
\text { Family_XIII } \\
\text { Lachnospiraceae } \\
\text { Lachnospiraceae } \\
\text { Lachnospiraceae } \\
\text { Lachnospiraceae } \\
\text { Lachnospiraceae } \\
\text { Lachnospiraceae } \\
\text { Lachnospiraceae } \\
\text { Lachnospiraceae } \\
\text { Lachnospiraceae } \\
\text { Ruminococcaceae } \\
\text { Ruminococcaceae } \\
\text { Ruminococcaceae } \\
\text { Ruminococcaceae } \\
\text { Ruminococcaceae } \\
\text { Ruminococcaceae } \\
\text { Ruminococcaceae } \\
\text { Desulfuromonadaceae } \\
\text { Akkermansiaceae }\end{array}$ & $\begin{array}{l}\text { Phylum } \\
\text { Actinobacteria } \\
\text { Bacteroidetes } \\
\text { Bacteroidetes } \\
\text { Bacteroidetes } \\
\text { Bacteroidetes } \\
\text { Bacteroidetes } \\
\text { Bacteroidetes } \\
\text { Firmicutes } \\
\text { Firmicutes } \\
\text { Firmicutes } \\
\text { Firmicutes } \\
\text { Firmicutes } \\
\text { Firmicutes } \\
\text { Firmicutes } \\
\text { Firmicutes } \\
\text { Firmicutes } \\
\text { Firmicutes } \\
\text { Firmicutes } \\
\text { Firmicutes } \\
\text { Firmicutes } \\
\text { Firmicutes } \\
\text { Firmicutes } \\
\text { Firmicutes } \\
\text { Firmicutes } \\
\text { Firmicutes } \\
\text { Proteobacteria } \\
\text { Verrucomicrobia }\end{array}$ \\
\hline
\end{tabular}

C

$\square$ Control $\square$ DSS

$95 \%$ confidence intervals

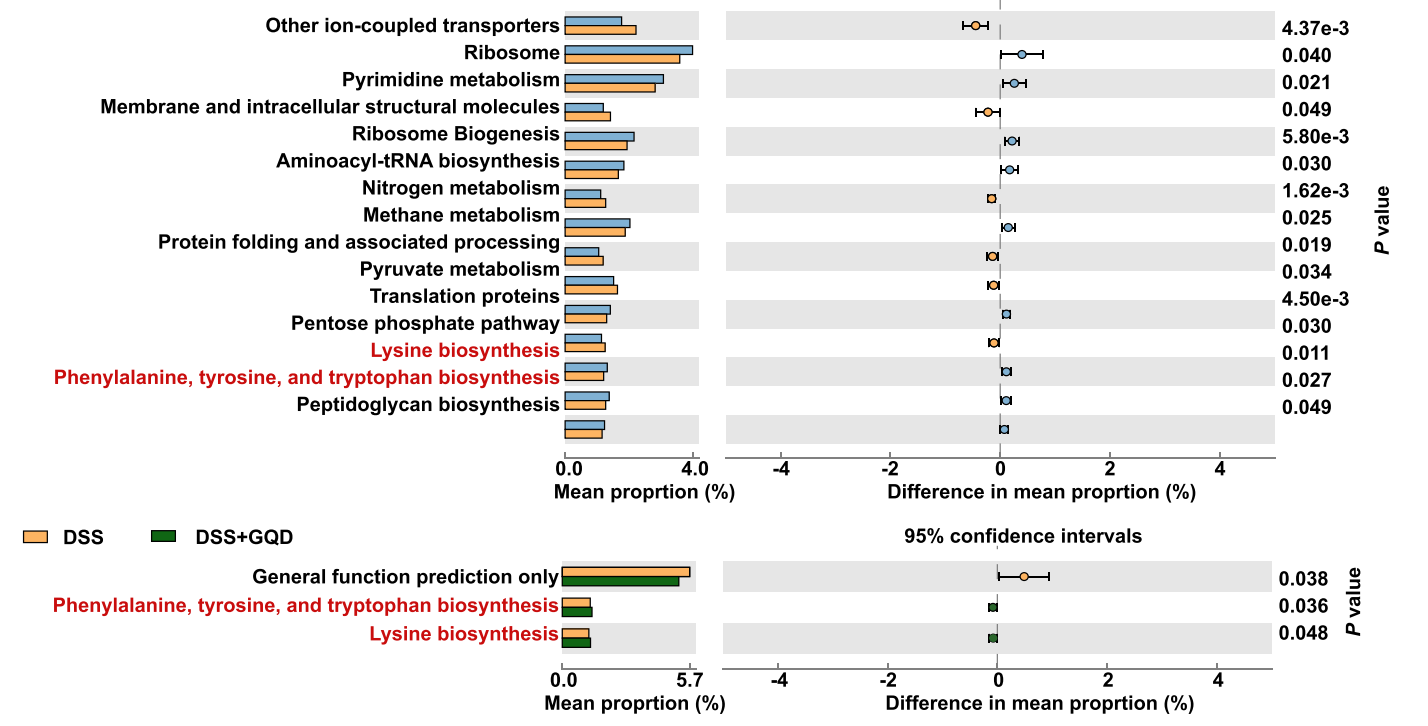

Figure 4 Differentially abundant microbes in the feces among different groups. (A) Differentially abundant genera identified by LEfSe with a linear discriminant analysis (LDA) score (threshold $\geq 2$ ). (B) The bubble matrix shows the average relative abundances of these genera, families, and phyla. The red font represents the bacteria that changed significantly in mice with colitis before and after GQD intervention. (C) Differentially abundant microbial gene functions predicted by PICRUSt compared by STAMP (version 2. I.3). The red font represents the pathway that changed significantly in mice with colitis before and after GQD intervention. $n=4-5$. 
lung pathological scores in GQD group were lower than SASP (Figure 5B, $P<0.05$ ). No significant change was seen between DSS+SASP and DSS groups (Figure 5B, $P>0.05$ ).

Next, we determined the lung function of mice using the Flexivent system. Compared to controls, Ers was significantly increased (Figure 5D, $P<0.01$ ), but Crs and Cst were both reduced (Figure 5E and $\mathrm{I}, P<0.05$ ) in colitis mice. No significant impact was observed on Rrs, Rn, G, and H (Figure 5C, $P>0.05$ ). On the contrary, the GQD treatment also significantly reversed the above indicators (Figure 5D and E, and $\mathrm{I}, P<0.05$ ). Significant differences existed between GQD and SASP interventions in Ers and Cst (Figure 5C-I, $P<0.05$ ), with no change in the lung function between DSS+SASP and DSS groups (Figure 5C-I, $P>0.05$ ). These findings indicated that GQD contributed more to alleviating the pulmonary inflammatory responses and improved the lung function of DSS-induced colitis mice than the SASP administration.

\section{GQD Decreases Inflammatory Myeloid Cells and Down-Regulates Related Cytokines in the Lung of DSS-Induced Colitis Mice}

Then, we examined the effect of GQD on pro-inflammatory myeloid cells in the lung of DSS-induced colitis mice. Compared with controls, the proportion of neutrophils was significantly increased in colitis mice (Figure 6A and $\mathrm{B}, P<$ 0.001 ), but no obvious change was presented in macrophages (Figure 6A and $\mathrm{C}, P>0.05$ ). The proportion of inflammatory monocytes was also increased in colitis mice compared with controls (Figure 6A and D, $P<0.01$ ), but no significant change in resident monocytes (Figure 6A and E, $P>0.05$ ). Consistent with changes in colonic tissue, GQD could decrease the accumulation of neutrophils and inflammatory monocytes (Figure $6 \mathrm{~A}, \mathrm{~B}$ and $\mathrm{D}, P<0.05$ ). However, no obvious change was seen after the SASP intervention.

On the other hand, in line with the results of colon tissue, the significantly increased levels of TNF- $\alpha$, IL- $1 \beta$, and IL-6 were observed in the lung of colitis mice (Figure 6F-H, $P<0.01$ ). As shown in Figure 6I-K, the relative expressions of CCR2 and CCL2 were increased significantly in colitis models $(P<0.05)$ with no change in the M-CSF expression $(P>$ 0.05). However, except for M-CSF $(P<0.05)$, the mRNA levels of these cytokines and chemokines were markedly down-regulated by GQD $(P<0.05)$. Only IL-1 $\beta$ expression was down-regulated by SASP $(P<0.05)$. Altogether, these outcomes demonstrated that GQD could inhibit the recruitment of inflammatory myeloid cells into the lung tissue and improve lung inflammation in mice with colitis.

\section{GQD Alleviates the Lung Microbiota Imbalance of DSS-Induced Colitis Mice}

We further explored whether the GQD administration could improve the lung microbiota imbalance in DSS-induced colitis mice. No significant difference in the alpha diversities as well as community structures was observed among control, DSS, and DSS $+\mathrm{GQD}$ groups (Figure 7A and B, $P>0.05$ ). However, the community composition significantly differed among the three groups (Figure 7C). LEfSe analysis identified that the genera Coprococcus_2 and Ochrobactrum exhibited higher relative proportions in the BALF of colitis mice than controls (Figure 8A and B). Whereas, the GQD administration strongly reduced the growth of these two genera (Figure $8 \mathrm{~A}$ and $\mathrm{B}$ ), implicating that they might serve as pulmonary microbial biomarkers in response to the GQD administration.

\section{Specific Microbes Affected by GQD Exhibits Significant Correlations to the Colonic and Lung Inflammation}

Next, we observed significant correlations between differentially abundant microbes and inflammatory cytokines in the colon (Figure 9A). In particular, the fecal genus Ruminococcaceae_UCG-013, exhibiting lower abundances of colitis mice than controls, were negatively correlated to colonic CCL2, CCR2, IL-6, TNF- $\alpha$, and IL-1 $\beta(P<0.05)$. Nevertheless, [Eubacterium]_fissicatena_group and Akkermansia enriched in colitis mice were positively associated with these above parameters $(P<0.05)$. Parabacteroides also showed similar significant trends to positively correlate with IL-1 $\beta(P=$ 0.085). On the other hand, the specific microbes in the BALF including Coprococcus_2 and Ochrobactrum, more abundant in colitis mice than controls, were positively linked to pulmonary CCL2, CCR2, IL-6, TNF- $\alpha$, and IL-1 $\beta$ (Figure 9B, $P<0.05$ ). Besides, Coprococcus_2 and Ochrobactrum in the lung positively connected with [Eubacterium] 
A
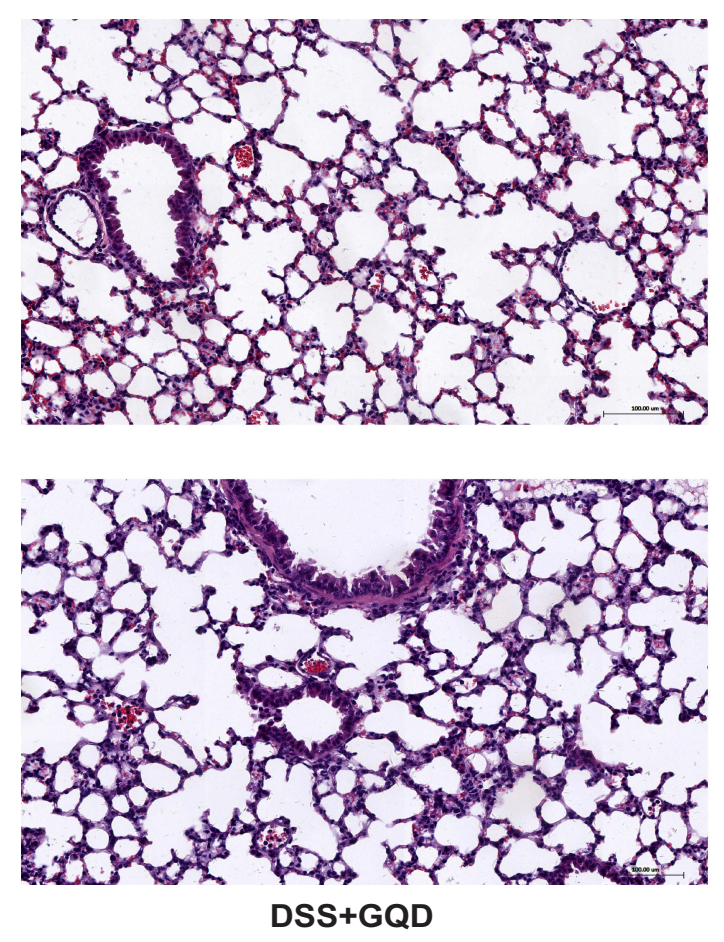

B

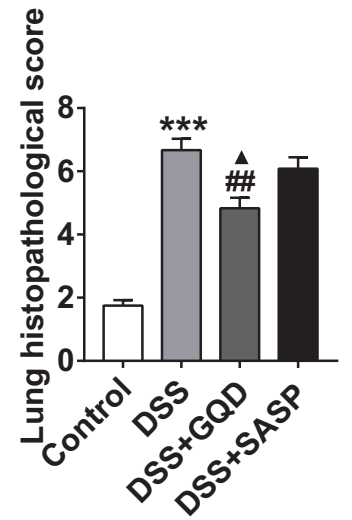

F

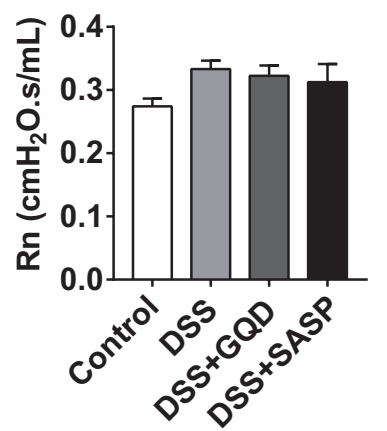

C

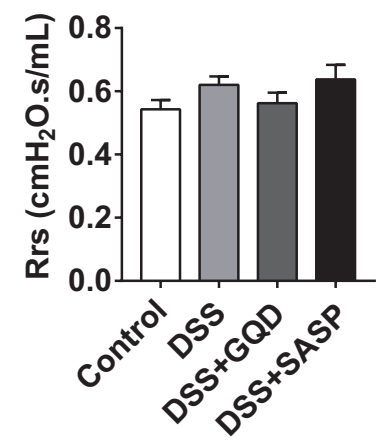

G

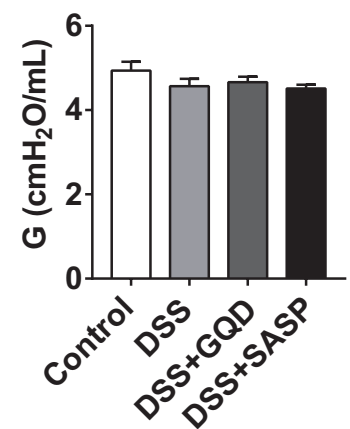

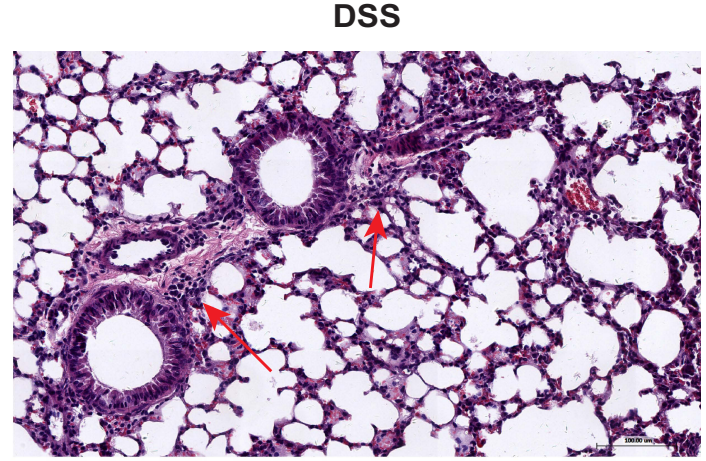

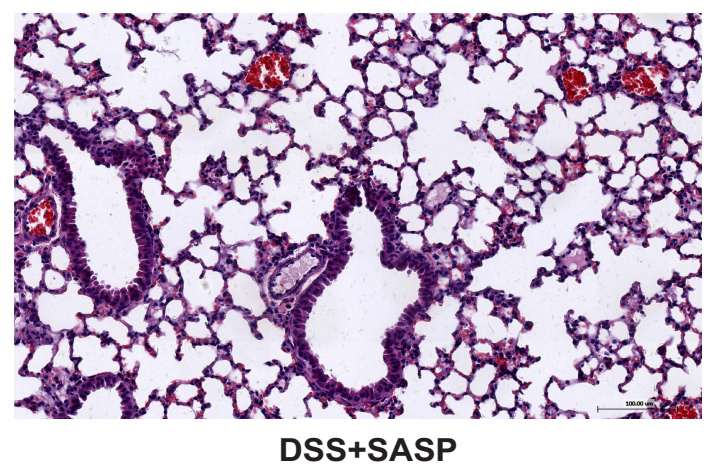

D

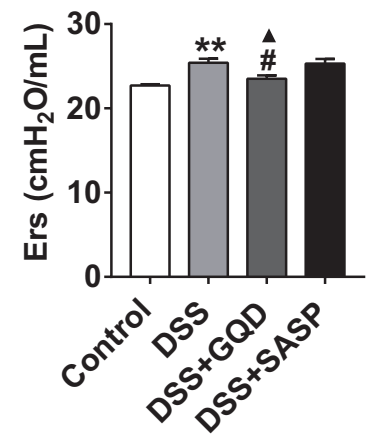

E

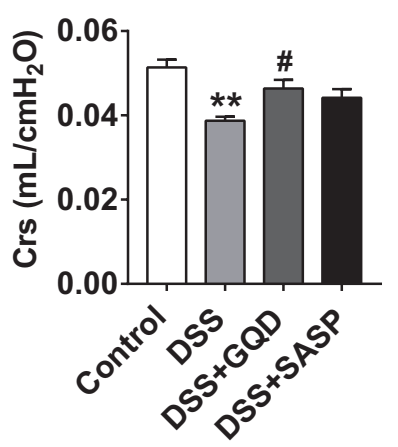

H

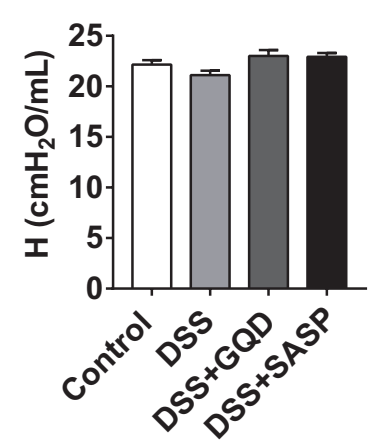

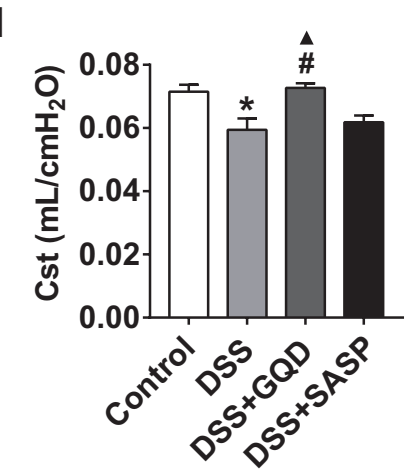

Figure 5 GQD alleviates pulmonary inflammation and improves lung function in DSS-induced colitis mice. (A) Lung pathological slides by the H\&E staining (200x). The red arrow means the site of inflammation. (B) Quantitative analysis of lung histopathology. (C-I) Comparison of the parameters involved in lung function of colitis mice. Mice were connected to the FlexiVent (SCIREQ) system for forced oscillations measurements. Respiratory system resistance, Rrs (C), elasticity resistance, Ers (D), compliance, Crs (E), the resistance of the central airways, Rn (F), tissue damping, $G(\mathbf{G})$, issue elastance, $H(\mathbf{H})$, and static compliance, Cst (I) were measured using a prime8 perturbation. Data were expressed as mean \pm SEM $(n=4-8)$. ${ }^{*} P<0.05$, ${ }^{* *} p<0.01$, ${ }^{* * *} P<0.001$, compared with the control group; ${ }^{\#} P<0.05$, ${ }^{\# \#} P<0.01$, compared with the DSS group; ${ }^{\wedge} P<0.05$, compared with the SASP group. 
A
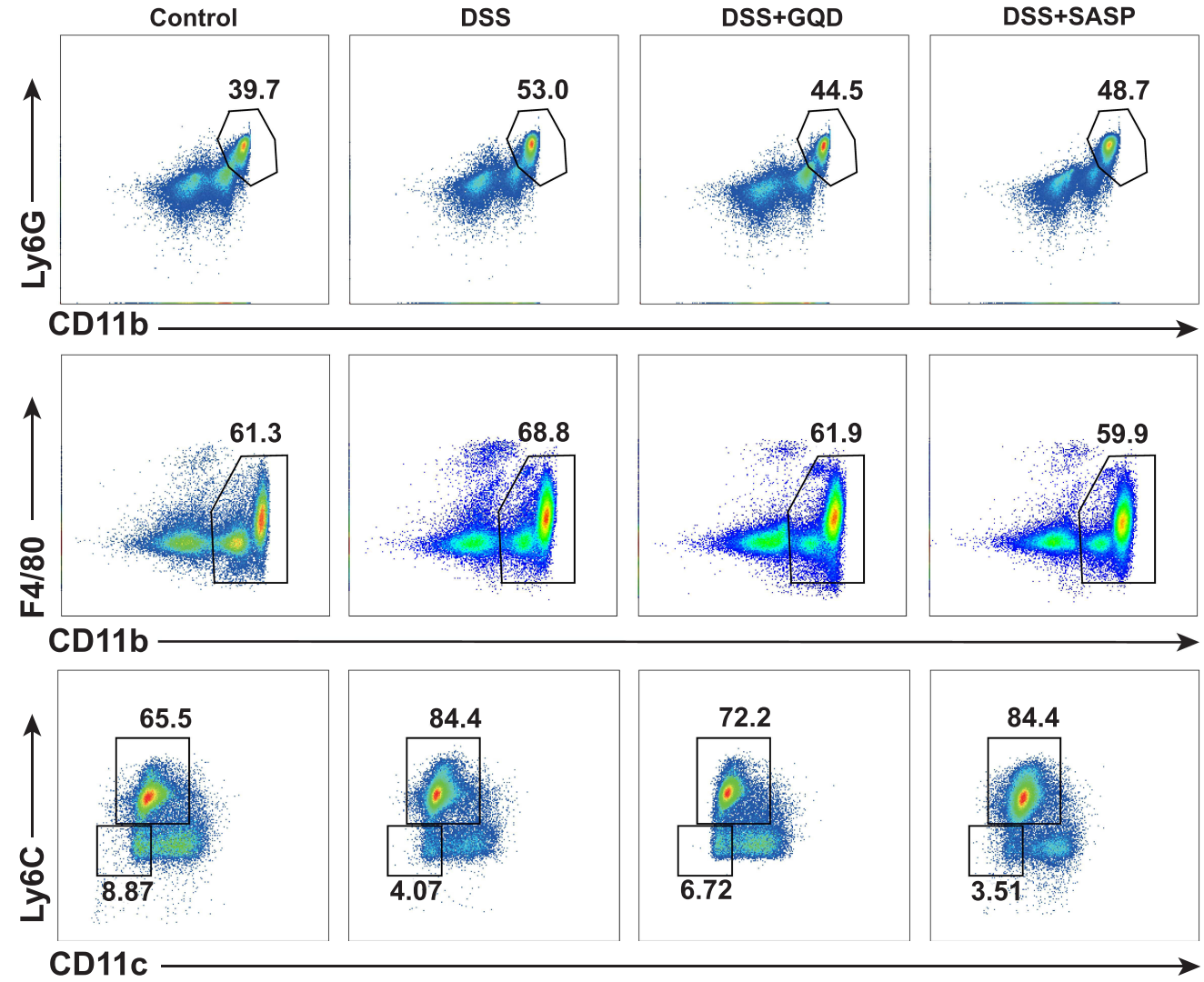

B

C Neutrophils Macrophages

D

E

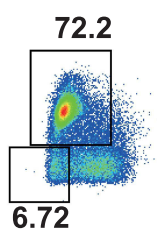
Monocytes (Inflam) Monocytes (Res)
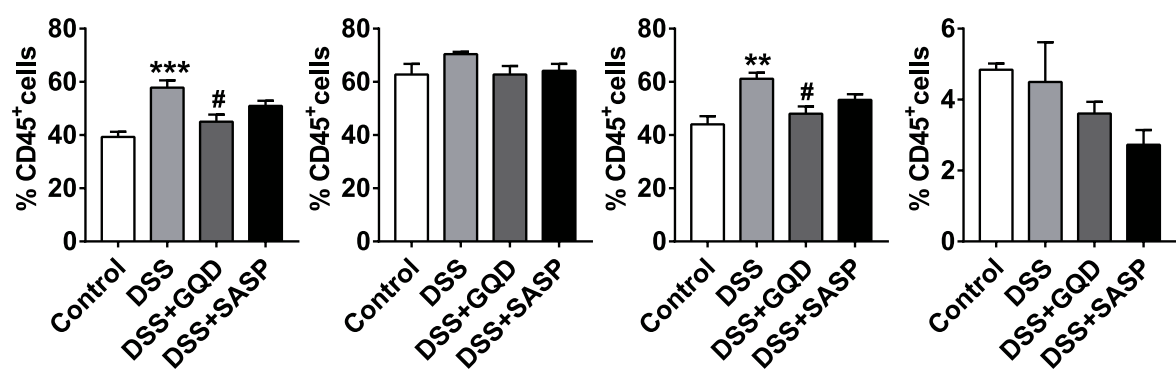

$\mathbf{F}$
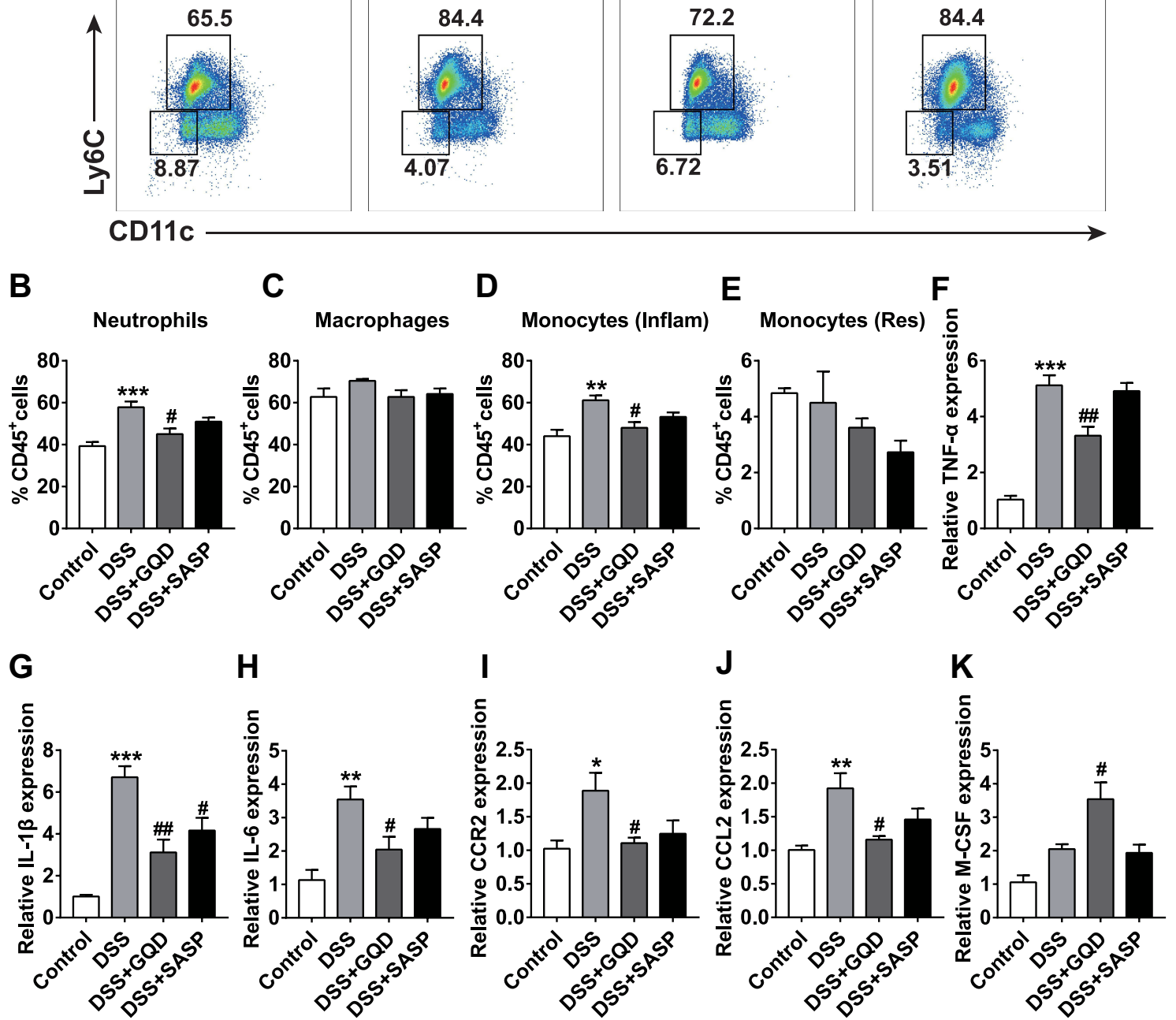

Figure 6 Myeloid cell populations and cytokines in the lung among different groups. (A) Representative gating strategies for identifying myeloid cells in the lung quantified by

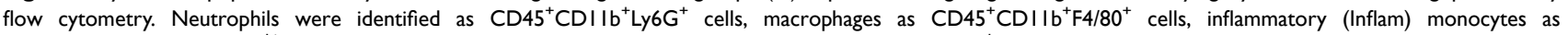

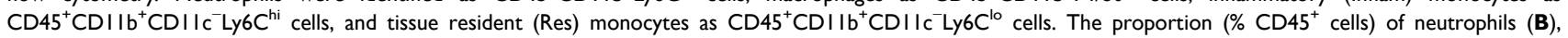
macrophages (C), inflammatory (Inflam) monocytes (D), and monocytes (Res) (E). The mRNA relative expressions of inflammatory cytokines including TNF- $\alpha$ (F), IL-I $\beta$ (G), IL-6 (H), CCR2 (I), CCL2 (J), and M-CSF (K) in pulmonary tissues assessed by RT-qPCR. Data were expressed as mean \pm SEM ( $\mathrm{n}=4-8)$. *P < 0.05 , **P < 0.01 , *** $P<$ 0.00 I, compared with the control group; ${ }^{\#} P<0.05$, ${ }^{\#} P<0.0$ I, compared with the DSS group. 
A
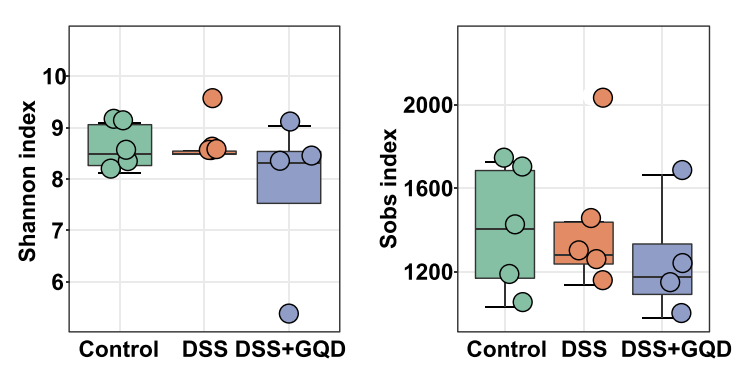

C

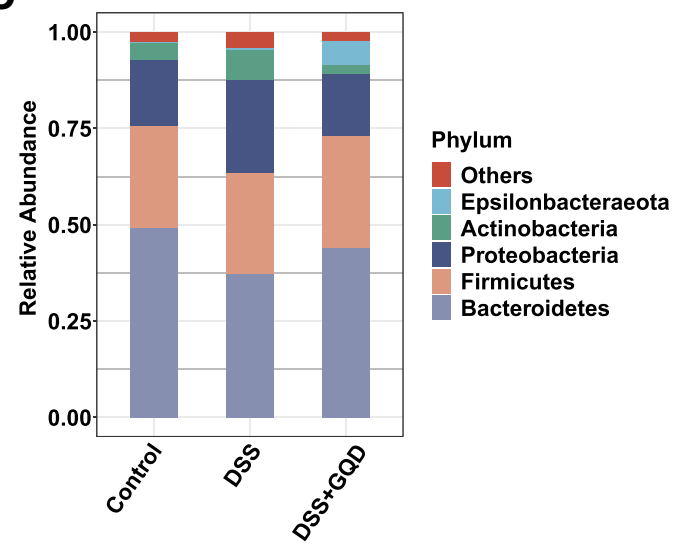

B
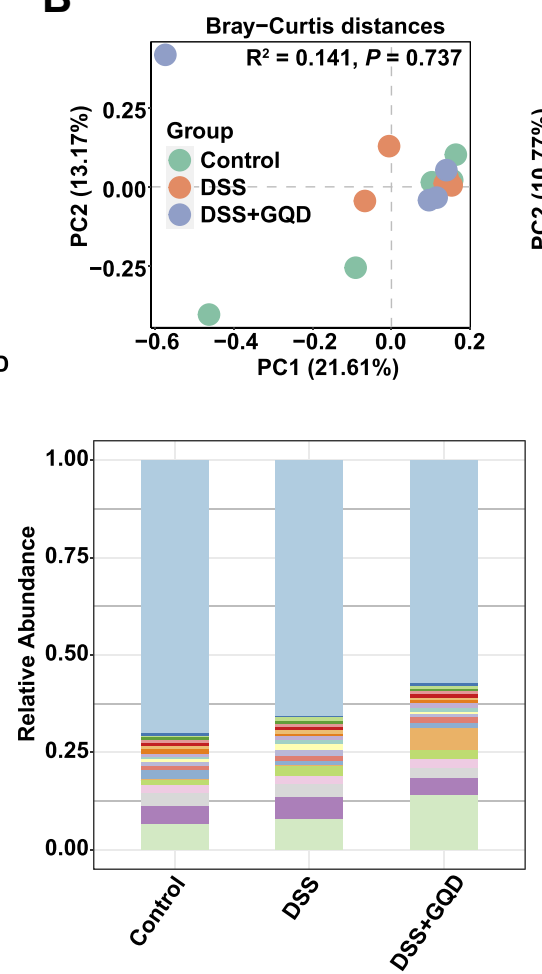

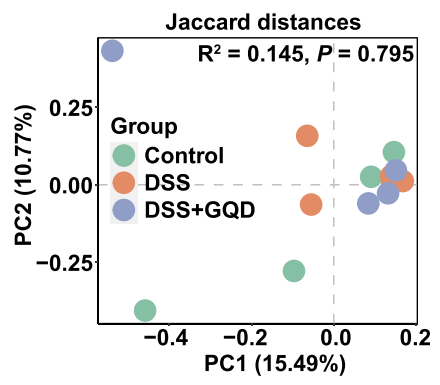

Genus

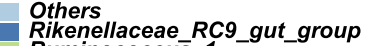

Ruminococcus__1

Aquabacterium

Prevotellaceae UCG-00

Prevotellaceae_UCG-001

Bifidobacterium

Blautia

Parabacteroides

Ambiguous_taxa

Roseburia

Lactobacillus

Alistipes

Escherichia-Shigella

Faecalibacterium

Lachnospiraceae_NK4A136 group

Pelomonas Bacteroides

Figure 7 The alterations in the pulmonary microbiota structure of DSS-induced colitis mice. (A) Shannon and sobs index. (B) Principal coordinate analysis (PCoA) plots based upon Bray-Curtis distances and Jaccard distances. (C) Abundant phyla and genera. $\mathrm{n}=4-5$.

fissicatena_group, Akkermansia, and Parabacteroides, but negatively correlated with Ruminococcaceae_UCG-013 in the intestine (Figure 9C, $P<0.05$ ).

Notably, macrophages, inflammatory monocytes, and neutrophils in the colon are positively linked to [Eubacterium] fissicatena_group but negatively associated with Ruminococcaceae_UCG-013 (Figure 9D, $P<0.01$ ). Moreover, Parabacteroides are also positively connected with macrophages (Figure 9D, $P<0.01$ ). No significant relationship was observed between pulmonary microbes and immune cells $(P>0.05)$.

These results implicated that the alterations in pulmonary and colonic inflammatory status of colitis mice might be partly mediated by their symbiotic microorganisms.

\section{Discussion}

The effector immune cells such as neutrophils, macrophages, inflammatory monocytes play an important regulatory role during UC. ${ }^{17,20}$ When UC occurs, these immune cells can infiltrate the colonic mucosa of UC patients and release plentiful pro-inflammatory cytokines such as IL-1 $\beta$, IL-6, and TNF- $\alpha$ to mediate the progress of UC inflammation. ${ }^{36}$ Besides, the critical chemokines including CCL2 and CCR2 were also highly expressed in UC patients. ${ }^{36,37}$ Our observations showed consistent alterations in the above parameters in colitis mice. Interestingly, the GQD intervention significantly reversed these alterations.

DSS as an external stimulus destroys barrier integrity and activates neutrophils, and then the accumulative recruitment of neutrophils further disrupts the intestinal mucosal barrier. ${ }^{38,39}$ GQD might repair the impaired colonic mucosal barrier by inhibiting the neutrophil recruitment to colonic tissue of DSS-induced colitis mice. A previous study has found that the number of macrophages in the colon mucosa of patients with UC is significantly higher than that of the normal population. ${ }^{40}$ In this study, GQD was found to decrease colonic macrophages and Ly6 $\mathrm{C}^{\text {hi }}$ inflammatory monocytes in mice with colitis. Currently, Ly6C ${ }^{\text {lo }}$ monocytes are believed to clear necrotic endothelial cells in the vascular system and repair vascular endothelial damage, acting as "macrophages of the circulatory system" and rarely entering intestinal tissues. ${ }^{41}$ In the case of 
A

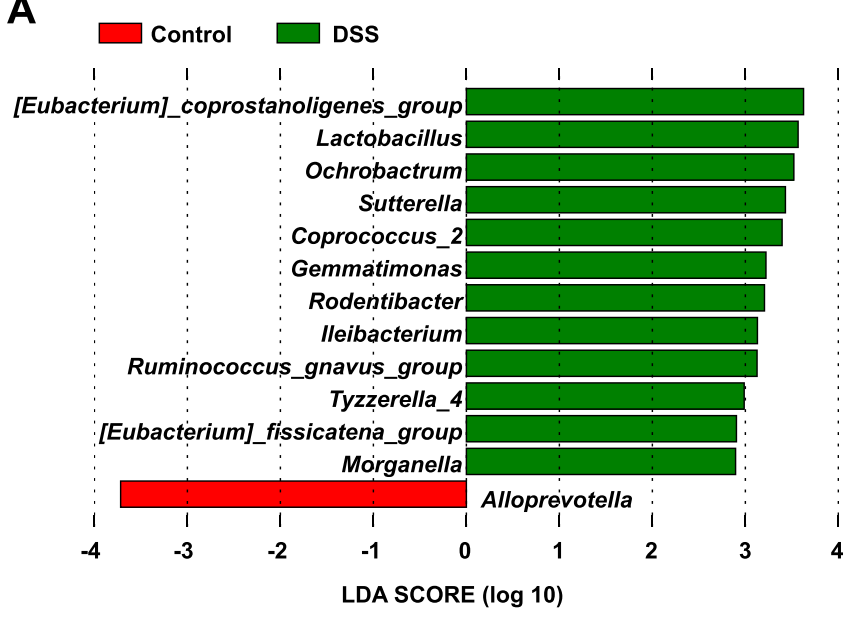

DSS DSS+GQD

B

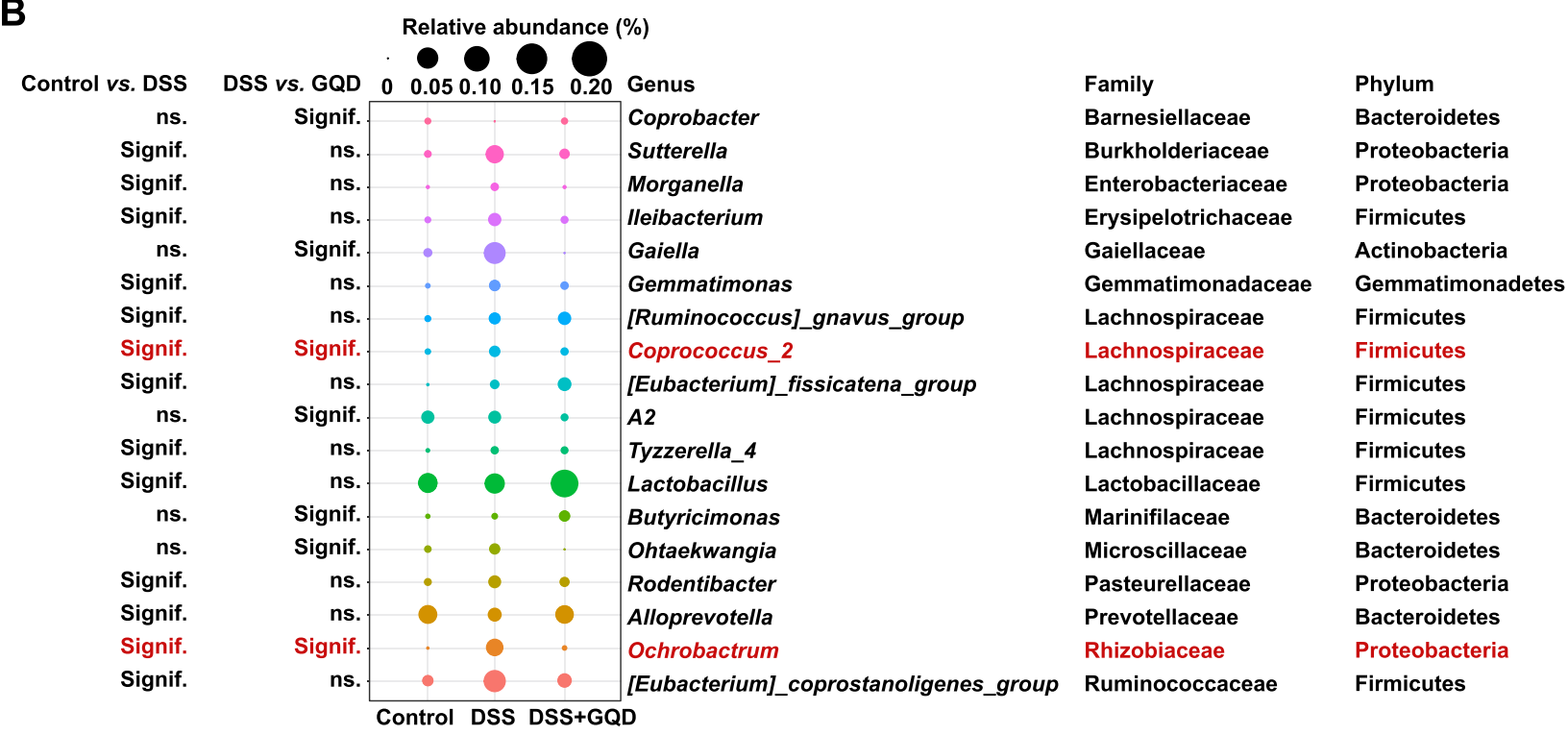

Figure 8 Differentially abundant microbes in the BALF among different groups. (A) Differentially abundant genera identified by LEfSe with a linear discriminant analysis (LDA) score (threshold $\geq 2$ ). (B) The bubble matrix shows the average relative abundances of these genera, families, and phyla. The red font represents the bacteria that changed significantly in mice with colitis before and after GQD intervention.

acute injury or infection, Ly6 $\mathrm{C}^{\text {hi }}$ monocytes differentiate into Ly $6 \mathrm{C}^{\mathrm{lo}}$ monocytes to replace macrophages to repair tissue. ${ }^{42}$ CCL2 and CCR2 are the key mediators in the infiltration of monocytes for inflammation. ${ }^{43} \mathrm{Ly} 6 \mathrm{C}^{\text {hi }}$ inflammatory monocytes are recruited into inflamed areas and secretes a large number of pro-inflammatory cytokines, which aggravates the inflammatory response. ${ }^{44}$ Our data also showed that GQD inhibited the recruitment of Ly6C $\mathrm{C}^{\text {hi }}$ inflammatory monocytes in the colon tissue of DSS colitis mice, perhaps by inhibiting the expression of CCR2 and CCL2.

Secondary pulmonary lesions are frequent in $\mathrm{IBD}^{3,45}$ and inflammatory cell infiltrates around the trachea and blood vessels have also been observed in lung biopsies in DSS-treated mouse models. ${ }^{46}$ In our study, the Crs of UC model mice, especially Cst, was decreased significantly, which may be related to the increase of the overall respiratory system elasticity resistance (Ers). Increased inspiratory or expiratory resistance causes the lung tissue to become less dilatable, resulting in a reduction in lung compliance. ${ }^{47}$ However, GQD could not only relieve lung damage, but also restore lung compliance, reduce elastic resistance, and reshape lung structure.

Based on much existing evidence, ${ }^{48-51}$ we believed that the repairment in the lung derived from the GQD medication might be related to the inhibition of inflammatory cell recruitment. Besides intestinal disease, CCL2 and CCR2 are also key factors of monocyte/macrophage recruitment in acute lung injury or pulmonary fibrosis. ${ }^{52,53}$ In colitis mice, however, 
A

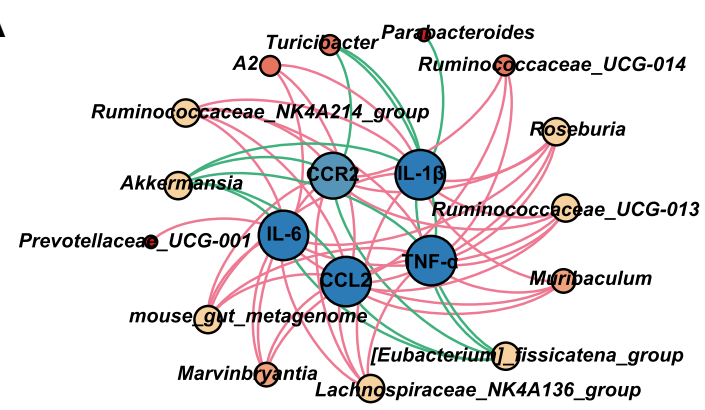

B

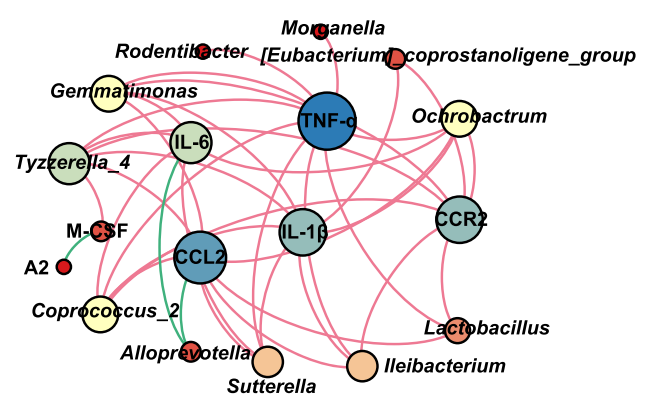

C
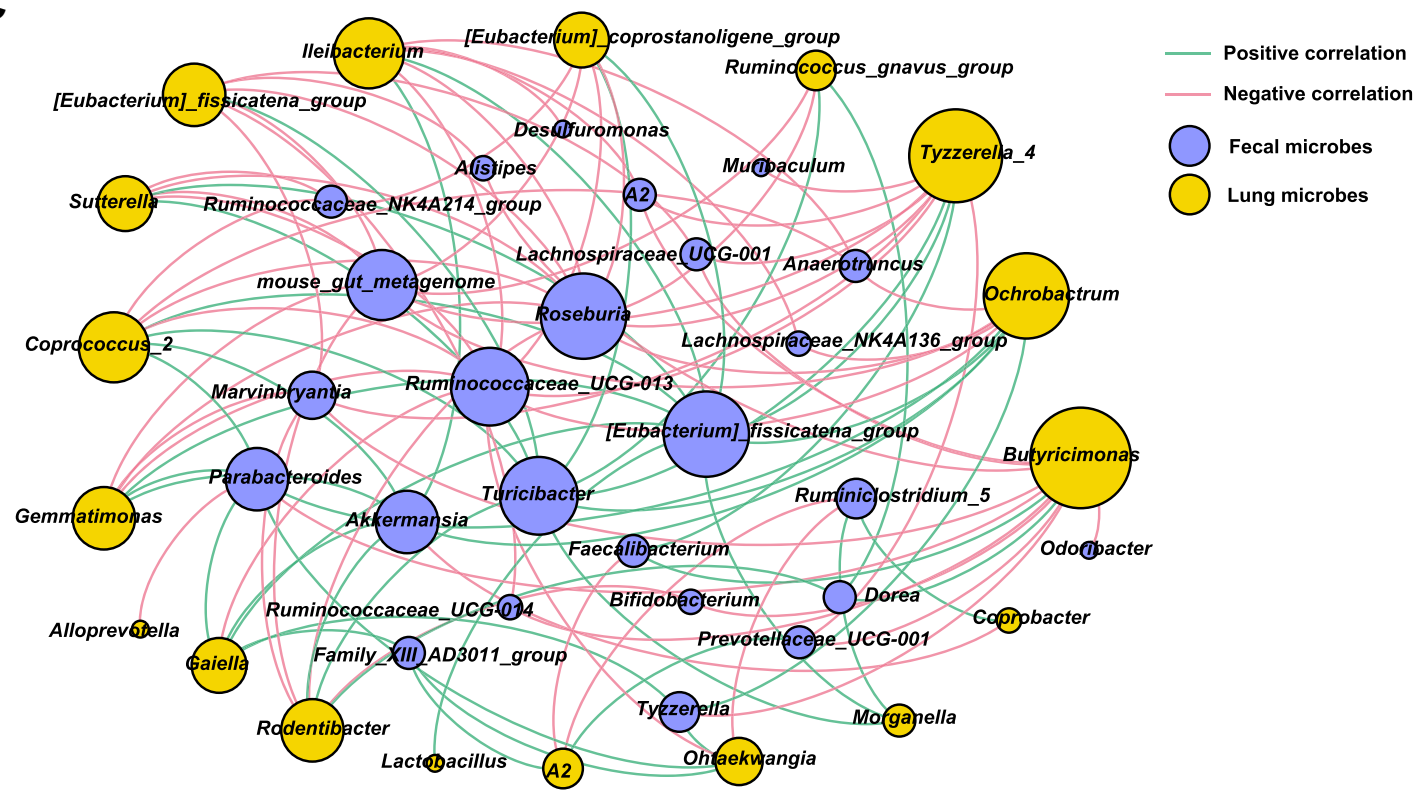

D
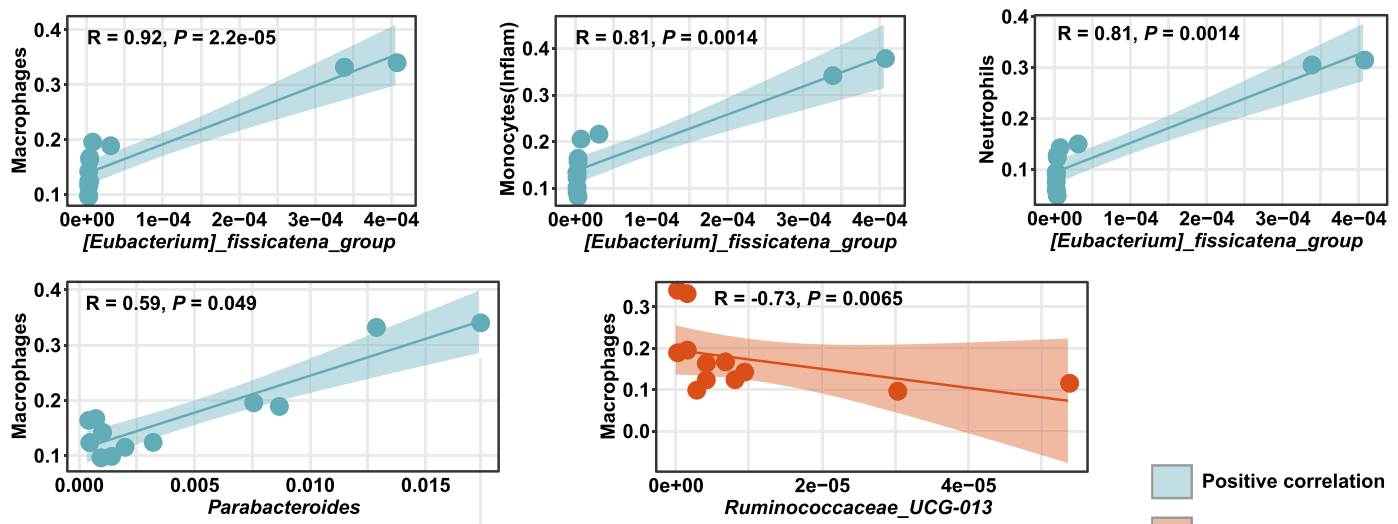

Positive correlation
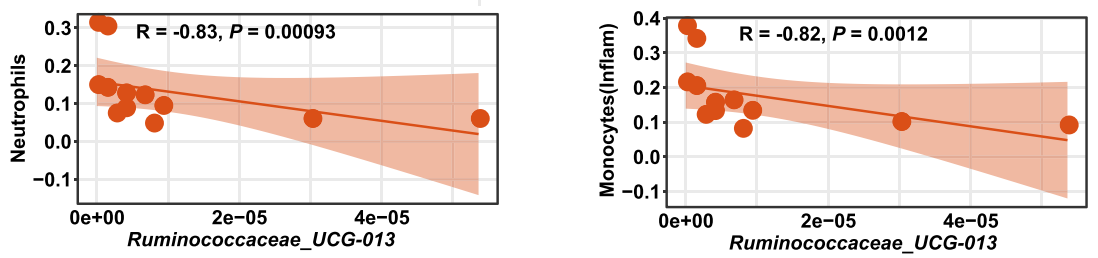

Negative correlation

Figure 9 Correlation of specific microbes with inflammation responses affected by DSS and GQD treatments. Network plots of differentially abundant genera (represented by nodes) that are significantly associated with inflammatory cytokines and chemokines in the colon (A) and lung (B). (C) Network plot of the correlations between colonic and lung key microbes. Spearman correlation coefficients were determined using the "psych" package in R (version 3.3.I). A positive correlation between nodes is indicated by green connecting lines, but a negative correlation by pink. Spearman correlations with the $P$ value of more than 0.05 were not shown. Gephi (version 0.9 .2 ) was used to visualize these network graphs. (D) Significant associations between key genera and immune cells in the colon were calculated by Spearman correlation pipeline. No significant connections were observed between microbes and immune cells in the lung. 
IL-6 mediates the recruitment of circulating neutrophils to the lung. ${ }^{54}$ Similarly, our results showed the accumulation of above immune cells and cytokines in the lung of colitis mice. Surprisingly, GQD decreased the appearance of pulmonary neutrophils and inflammatory monocytes of mice with colitis, along with the down-regulated expressions of CCR2, CCL2, and IL-6. Interestingly, the SASP administration showed similar influences on colonic inflammation as GQD but little effect on lung injury. This suggested that GQD acts as a multi-target regulatory formula targeting diseased colon and colon-related vital organs, including the lung, relative to the SASP medication used as a targeted therapy for the colonic site.

On the other hand, there is an intimate correlation between intestinal microbiota and the incidence of UC. In this study, we observed that Parabacteroides, [Eubacterium]_Fissicatena_group, Ruminococcaceae, and Akkermansia were differentially abundant intestinal microbes after the treatment with GQD. In line with previous reports, the acute exacerbations of UC were accompanied by the increased number of Parabacteroides as compared to the remission stage. ${ }^{55}$ [Eubacterium] _Fissicatena_group, exhibiting the same change with Parabacteroides, has a high correlation with the occurrence of obesity and related metabolic disorders. Moreover, the inflammatory environment created by obesity has even been suggested to accelerate the deterioration of UC. ${ }^{56,57}$ In contrast, less abundance of Ruminococcaceae_UCG-013 occurred in colitis mice than controls as published researches. ${ }^{58-60}$ Interestingly, our data showed that the genera Akkermansia was increased in DSS-induced colitis mice, which appeared to contradict many recent studies. Of note, the pro-inflammatory or anti-inflammatory effects of Akkermansia remain controversial on IBD. ${ }^{61}$ This contradictory finding might be related to the different mouse models used, including Cyp27b1, Hes1, and IL-10 ${ }^{-/}$, as well as DSS-induced colitis. ${ }^{62,63}$ Whether Akkermansia promotes or inhibits the development of intestinal mucosal inflammation needs further exploration. As expected, the addition of GQD significantly restored the gut microbiota imbalance by reversing the alterations of the above specific bacteria, thus contributing to a prominent improvement in experimental colitis.

The host and resident microbiota are closely connected by microbial gene functions. In this study, our results demonstrated that GQD reversed the decreases in the microbial genes associated with lysine biosynthesis as well as phenylalanine, tyrosine, and tryptophan biosynthesis in the intestines of colitis mice compared to controls. In general, with the involvement of these two pathways, intestinal bacteria could produce numerous metabolites such as indoxyl sulfate and p-Cresol sulfate. ${ }^{64}$ Afterwards, these microbial byproducts exert protective effects on intestinal barrier function and antioxidant activity of the host. ${ }^{64}$ Future studies are desired to explore whether these microbial metabolic pathways could be potential targets for GQD to repair colitis by modulating the gut microbiota.

The bidirectional cross-talk within the gut-lung axis has been extensively reported in recent years. ${ }^{10}$ Intestinal microorganisms can stimulate immune cells to release cytokines into the blood, mediating systemic inflammatory responses. ${ }^{36,65}$ These cytokines and microbial metabolites can bind to receptors on the surface of lung epithelial cells to activate lung epithelial cells and release more inflammatory biological factors into the lungs. ${ }^{66-69}$ The alterations of the pulmonary microbial community would also aggravate lung inflammation. ${ }^{67}$ Beyond alterations in the gut microbiota, we also observed the dramatic changes of pulmonary microbes in colitis mice, indirectly confirming the cross-talk between gut and lung. As reported, ${ }^{46}$ the bacterial load was increased in mouse lungs and therefore induced pulmonary inflammation in colitis mice. Here, we have also found significant variation in the lung microbiome of colitis mice such as Ochrobactrum and Coprococcus_2 after GQD administration. Ochrobactrum and Coprococcus_2 are positively correlated with a variety of infectious diseases such as tuberculosis and represent significant resistance to broad-spectrum antibiotics that may reduce the efficacy. ${ }^{70-73}$ It is gratifying that GQD could inhibit the colonization of the above two microbes to improve the imbalance of pulmonary microbiota induced by UC.

Interestingly, we further observed significant association between host immunity and commensal microbes associated with GQD intervention. Moreover, significant correlations between pulmonary and intestinal specific microbes affected by GQD, combined with simultaneous alterations in inflammatory responses between colon and lung, further illustrated the potential role of the gut-lung axis in the pathogenesis of UC. Collectively, colonic and lung inflammatory responses in UC might be partially modulated by the resident microbiota along the gut-lung axis, which needs further proof in our subsequent trials. 


\section{Conclusion}

In summary, our work shows that the oral administration of GQD could ameliorate the DSS-induced colitis and its derived lung inflammation related to inhibiting the recruitment of inflammatory myeloid cells (eg, neutrophils, macrophages, and inflammatory monocytes) and the secretion of pro-inflammatory biomarkers (eg TNF- $\alpha$, IL-1 $\beta$, IL-6, CCR2, and CCL2). On the other hand, GQD could improve colitis-associated intestinal and lung microbiota imbalance via modulating the colonization of specific microbes. These microbes mainly include Ruminococcaceae_UCG-013, [Eubacterium]_fissicatena_group, and Akkermansia in the colon, as well as Coprococcus_2 and Ochrobactrum in the lung. Moreover, these GQD-associated microbes might be involved in the deterioration of colonic and pulmonary inflammation as their significant associations with GQD-associated cytokines or even inflammatory cells. More importantly, prominent correlations between GQD-associated specific intestinal and lung microbes implicate that the bidirectional cross-talk within the gut-lung axis might exist and mediate the progress and recovery of inflammation in colitis mice. Further research will emphasize the interaction loop between GQD-associated specific microorganisms and immune cells based on the gut-lung axis during the pathophysiology of UC.

\section{Abbreviations}

UC, ulcerative colitis; IBD, inflammatory bowel disease; CD, Crohn's disease; DSS, dextran sulfate sodium; TCM, traditional Chinese medicine; GQD, Gegen Qinlian Decoction; DW, distilled water; SASP, sulfasalazine; DAI, disease activity index; H\&E, hematoxylin and eosin; Rrs, respiratory system resistance; Ers, elasticity resistance; Crs, compliance; Rn, the resistance of the central airways; G, tissue damping; $\mathrm{H}$, tissue elastance; Cst, static compliance; BALF, bronchoalveolar lavage fluid; OTUs, operational taxonomic units; PCoA, principal coordinates analysis; PERMANOVA, permutational multivariate analysis of variance; LEfSe, discriminant analysis (LDA) effect size; FDR, false discovery rate; SEM, standard error of the mean.

\section{Data Sharing Statement}

The raw sequencing data supporting this study are available in the NCBI Sequence Read Archive (SRA) repository under accession number PRJNA785390. The remaining data that support the findings of this study are available from the corresponding author upon reasonable request.

\section{Acknowledgments}

This research was supported by the 13th Five-Year Plan for the National Key R\&D Program of China (No. 2018YFC1705405) and Fundamental Research Funds for Central Universities (No. 2019-JYB-TD014).

\section{Author Contributions}

All authors made a significant contribution to the work reported, whether that is in the conception, study design, execution, acquisition of data, analysis, and interpretation, or in all these areas; took part in drafting, revising, or critically reviewing the article; gave final approval of the version to be published; have agreed on the journal to which the article has been submitted; and agree to be accountable for all aspects of the work.

\section{Disclosure}

The authors report no conflicts of interest in this work.

\section{References}

1. Rubin DT, Ananthakrishnan AN, Siegel CA, Sauer BG, Long MD. ACG clinical guideline: ulcerative colitis in adults. Am J Gastroenterol. 2019;114 (3):384-413.

2. Zippi M, Corrado C, Pica R, et al. Extraintestinal manifestations in a large series of Italian inflammatory bowel disease patients. World $J$ Gastroenterol. 2014;20(46):17463-17467.

3. Massart A, Hunt DP. Pulmonary manifestations of inflammatory bowel disease. Am J Med. 2020;133(1):39-43.

4. Turner-Warwick M. Fibrosing alveolitis and chronic liver disease. $Q J$ Med. 1968;37(145):133-149.

5. Camus P, Piard F, Ashcroft T, Gal AA, Colby TV. The lung in inflammatory bowel disease. Medicine (Baltimore). 1993;72(3):151-183. 
6. Sato H, Okada F, Matsumoto S, et al. Chest high-resolution computed tomography findings in 601 patients with inflammatory bowel diseases. Acad Radiol. 2018;25(4):407-414.

7. Ates F, Karincaoglu M, Hacievliyagil SS, Yalniz M, Seckin Y. Alterations in the pulmonary function tests of inflammatory bowel diseases. Turk J Gastroenterol. 2011;22(3):293-299.

8. Kraft SC, Earle RH, Roesler M, Esterly JR. Unexplained bronchopulmonary disease with inflammatory bowel disease. Arch Intern Med. 1976 ; 136 (4):454-459.

9. Dang AT, Marsland BJ. Microbes, metabolites, and the gut-lung axis. Mucosal Immunol. 2019;12(4):843-850.

10. Enaud R, Prevel R, Ciarlo E, et al. The gut-lung axis in health and respiratory diseases: a place for inter-organ and inter-Kingdom crosstalks. Front Cell Infect Microbiol. 2020;10:9.

11. Tulic MK, Piche T, Verhasselt V. Lung-gut cross-talk: evidence, mechanisms and implications for the mucosal inflammatory diseases. Clin Exp Allergy. 2016;46(4):519-528.

12. Tulic MK, Vivinus-Nebot M, Rekima A, et al. Presence of commensal house dust mite allergen in human gastrointestinal tract: a potential contributor to intestinal barrier dysfunction. Gut. 2016;65(5):757-766.

13. Eksteen B, Grant AJ, Miles A, et al. Hepatic endothelial CCL 25 mediates the recruitment of CCR $9^{+}$gut-homing lymphocytes to the liver in primary sclerosing cholangitis. J Exp Med. 2004;200(11):1511-1517.

14. Leal MC, Dabritz J. Immunoregulatory role of myeloid-derived cells in inflammatory bowel disease. Inflamm Bowel Dis. $2015 ; 21(12): 2936-2947$.

15. Glennon-Alty L, Hackett AP, Chapman EA, Wright HL. Neutrophils and redox stress in the pathogenesis of autoimmune disease. Free Radic Biol Med. 2018;125:25-35.

16. Zhang T, Jiang JH, Liu JT, et al. MK2 is required for neutrophil-derived ROS production and inflammatory bowel disease. Front Med. $2020 ; 7: 207$.

17. Fournier BM, Parkos CA. The role of neutrophils during intestinal inflammation. Mucosal Immunol. 2012;5(4):354-366.

18. Li Q, Wang D, Hao S, et al. CD169 expressing macrophage, a key subset in mesenteric lymph nodes promotes mucosal inflammation in dextran sulfate sodium-induced colitis. Front Immunol. 2017;8:669.

19. Asano K, Takahashi N, Ushiki M, et al. Intestinal CD169(+) macrophages initiate mucosal inflammation by secreting CCL8 that recruits inflammatory monocytes. Nature Commun. 2015;6:1-4.

20. Jones GR, Bain CC, Fenton TM, et al. Dynamics of colon monocyte and macrophage activation during colitis. Front Immunol. $2018 ; 9: 2764$.

21. Nishida A, Inoue R, Inatomi O, Bamba S, Naito Y, Andoh A. Gut microbiota in the pathogenesis of inflammatory bowel disease. Clin $J$ Gastroenterol. 2018;11(1):1-10.

22. Huffnagle GB, Dickson RP, Lukacs NW. The respiratory tract microbiome and lung inflammation: a two-way street. Mucosal Immunol. 2017;10 (2):299-306.

23. Scales BS, Dickson RP, Huffnagle GB. A tale of two sites: how inflammation can reshape the microbiomes of the gut and lungs. $J$ Leukoc Biol. 2016;100(5):943-950.

24. Fan YL, Yi W, Huang H, Mei ZG, Feng ZT. Efficacy of herbal medicine (Gegen Qinlian Decoction) on ulcerative colitis: a systematic review of randomized controlled trials. Medicine. 2019;98:52.

25. Xu BL, Zhang GJ, Ji YB. Active components alignment of Gegenqinlian decoction protects ulcerative colitis by attenuating inflammatory and oxidative stress. J Ethnopharmacol. 2015;162:253-260.

26. Cui L, Feng L, Zhang ZH, Jia XB. The anti-inflammation effect of baicalin on experimental colitis through inhibiting TLR4/NF- $\kappa B$ pathway activation. Int Immunopharmacol. 2014;23(1):294-303.

27. Kwon HS, Oh SM, Kim JK. Glabridin, a functional compound of liquorice, attenuates colonic inflammation in mice with dextran sulphate sodium-induced colitis. Clin Exp Immunol. 2008;151(1):165-173.

28. Yan F, Wang L, Shi Y, et al. Berberine promotes recovery of colitis and inhibits inflammatory responses in colonic macrophages and epithelial cells in DSS-treated mice. Am J Physiol Gastrointest Liver Physiol. 2012;302(5):G504-514.

29. Liu X, Fan Y, Du L, Mei Z, Fu Y. In silico and in vivo studies on the mechanisms of Chinese medicine formula (Gegen Qinlian decoction) in the treatment of ulcerative colitis. Front Pharmacol. 2021;12:665102.

30. Ding Z, Zhong R, Yang Y, et al. Systems pharmacology reveals the mechanism of activity of Ge-Gen-Qin-Lian decoction against LPS-induced acute lung injury: a novel strategy for exploring active components and effective mechanism of TCM formulae. Pharmacol Res. 2020;156:104759.

31. Li Y, Liu J, Ma P, et al. Gegen Qinlian Tang regulates MMP-9/p38 MAPK pathway to repair intestinal mucosal barrier function in mice with ulcerative colitis. Chinese J Exp Tradit Med Formulae. 2021;27(4):8-15.

32. Dutton JW 3rd, Artwohl JE, Huang X, Fortman JD. Assessment of pain associated with the injection of sodium pentobarbital in laboratory mice (mus musculus). J Am Assoc Lab Anim Sci. 2019;58(3):373-379.

33. Sun Z, Li J, Dai Y, et al. Indigo naturalis alleviates dextran sulfate sodium-induced colitis in rats via altering gut microbiota. Front Microbiol. 2020;11:731.

34. Lai HC, Lin TL, Chen TW, et al. Gut microbiota modulates COPD pathogenesis: role of anti-inflammatory Parabacteroides goldsteinii lipopolysaccharide. Gut. 2021;71:322599.

35. Zhang W, Li Q, Li D, Li J, Aki D, Liu YC. The E3 ligase VHL controls alveolar macrophage function via metabolic-epigenetic regulation. J Exp Med. 2018;215(12):3180-3193.

36. Singh UP, Singh NP, Murphy EA, et al. Chemokine and cytokine levels in inflammatory bowel disease patients. Cytokine. 2016;77:44-49.

37. Popivanova BK, Kostadinova FI, Furuichi K, et al. Blockade of a chemokine, CCL2, reduces chronic colitis-associated carcinogenesis in mice. Cancer Res. 2009;69(19):7884-7892.

38. Pei LY, Ke YS, Zhao HH, et al. Role of colonic microbiota in the pathogenesis of ulcerative colitis. BMC Gastroenterol. 2019;19(1):10.

39. Jin S, Zhao D, Cai CW, et al. Low-dose penicillin exposure in early life decreases Th17 and the susceptibility to DSS colitis in mice through gut microbiota modification. Sci Rep. 2017;7:43662.

40. Yao D, Dong M, Dai C, Wu S. Inflammation and inflammatory cytokine contribute to the initiation and development of ulcerative colitis and its associated cancer. Inflamm Bowel Dis. 2019;25(10):1595-1602.

41. Varol C, Vallon-Eberhard A, Elinav E, et al. Intestinal lamina propria dendritic cell subsets have different origin and functions. Immunity. 2009;31 (3):502-512. 
42. Kratofil RM, Kubes P, Deniset JF. Monocyte conversion during inflammation and injury. Arterioscler Thromb Vasc Biol. 2017;37(1):35-42.

43. Raghu H, Lepus CM, Wang Q, et al. CCL2/CCR2, but not CCL5/CCR5, mediates monocyte recruitment, inflammation and cartilage destruction in osteoarthritis. Ann Rheum Dis. 2017;76(5):914-922.

44. Li QT, Wang D, Hao SY, et al. CD169 expressing macrophage, a key subset in mesenteric lymph nodes promotes mucosal inflammation in dextran sulfate sodium-induced colitis. Front Immunol. 2017;8:669.

45. Gupta SJ, Gupta VL, Kothari HG, Samarth AR, Gaikwad NR, Parmar SM. Assessment of occult pulmonary involvement in ulcerative colitis. Inflamm Intest Dis. 2020;5(3):144-150.

46. Liu G, Mateer SW, Hsuz A, et al. Platelet activating factor receptor regulates colitis-induced pulmonary inflammation through the NLRP3 inflammasome. Mucosal Immunol. 2019;12(4):862-873.

47. Nay MA, Auvet A, Mankikian J, Herve V, Dequin PF, Guillon A. Evaluation of a flexible bronchoscope prototype designed for bronchoscopy during mechanical ventilation: a proof-of-concept study. Anaesthesia. 2017;72(6):719-728.

48. Lokwani R, Wark PAB, Baines KJ, Barker D, Simpson JL. Hypersegmented airway neutrophils and its association with reduced lung function in adults with obstructive airway disease: an exploratory study. BMJ Open. 2019;9(1):e024330.

49. Abraham E. Neutrophils and acute lung injury. Crit Care Med. 2003;31(4 Suppl):S195-199.

50. Yamasaki K, Eeden SFV. Lung macrophage phenotypes and functional responses: role in the pathogenesis of COPD. Int J Mol Sci. 2018;19:2.

51. Zhang WK, Dai HJ, Lin F, et al. Ly-6C(high) inflammatory-monocyte recruitment is regulated by $\mathrm{p} 38 \mathrm{MAPK} / \mathrm{MCP}-1$ activation and promotes ventilator-induced lung injury. Int Immunopharmacol. 2020;78:106015.

52. Yang JB, Agarwal M, Ling S, et al. Diverse injury pathways induce alveolar epithelial cell CCL2/12, which promotes lung fibrosis. Am J Respir Cell Mol Biol. 2020;62(5):622-632.

53. Guillamat-Prats R, Camprubi-Rimblas M, Tantinya N, Artigas A. Local depletion of the recruitment of macrophages reduces acute lung injury in rats. Eur Respir J. 2018;52:PA4296.

54. Mateer SW, Mathe A, Bruce J, et al. IL-6 drives neutrophil-mediated pulmonary inflammation associated with bacteremia in murine models of colitis. Am J Pathol. 2018;188(7):1625-1639.

55. Walujkar SA, Kumbhare SV, Marathe NP, et al. Molecular profiling of mucosal tissue associated microbiota in patients manifesting acute exacerbations and remission stage of ulcerative colitis. World J Microbiol Biotechnol. 2018;34(6):76.

56. El-Dallal M, Stein DJ, Raita Y, Feuerstein JD. The impact of obesity on hospitalized patients with ulcerative colitis. Ann Gastroenterol. 2021;34 (2):196-201.

57. Song YJ, Shen HT, Liu TT, et al. Effects of three different mannans on obesity and gut microbiota in high-fat diet-fed C57BL/6J mice. Food Funct. 2021;12(10):4606-4620.

58. Vich Vila A, Imhann F, Collij V, et al. Gut microbiota composition and functional changes in inflammatory bowel disease and irritable bowel syndrome. Sci Transl Med. 2018;10:472.

59. Lo Presti A, Zorzi F, Del Chierico F, et al. Fecal and mucosal microbiota profiling in irritable bowel syndrome and inflammatory bowel disease. Front Microbiol. 2019;10:1655.

60. Yilmaz B, Juillerat P, Oyas O, et al. Microbial network disturbances in relapsing refractory Crohn's disease. Nat Med. $2019 ; 25(2): 323-336$.

61. Zhang T, Ji X, Lu G, Zhang F. The potential of Akkermansia muciniphila in inflammatory bowel disease. Appl Microbiol Biotechnol. 2021;105(1415):5785-5794.

62. Lee Y, Sugihara K, Gillilland MG 3rd, Jon S, Kamada N, Moon JJ. Hyaluronic acid-bilirubin nanomedicine for targeted modulation of dysregulated intestinal barrier, microbiome and immune responses in colitis. Nat Mater. 2020;19(1):118-126.

63. Zhu W, Yan J, Zhi C, Zhou Q, Yuan X. 1,25(OH) $)_{2} \mathrm{D}_{3}$ deficiency-induced gut microbial dysbiosis degrades the colonic mucus barrier in Cyp27b1 knockout mouse model. Gut Pathog. 2019;11(1):1-11.

64. Van Treuren W, Dodd D. Microbial contribution to the human metabolome: implications for health and disease. Annu Rev Pathol. 2020;15:345-369.

65. Martinez-Fierro ML, Garza-Veloz I, Rocha-Pizana MR, et al. Serum cytokine, chemokine, and growth factor profiles and their modulation in inflammatory bowel disease. Medicine. 2019;98:38.

66. Dumas A, Bernard L, Poquet Y, Lugo-Villarino G, Neyrolles O. The role of the lung microbiota and the gut-lung axis in respiratory infectious diseases. Cell Microbiol. 2018;20(12):e12966.

67. Leiva-Juarez MM, Kolls JK, Evans SE. Lung epithelial cells: therapeutically inducible effectors of antimicrobial defense. Mucosal Immunol. 2018;11(1):21-34

68. Zhang DP, Li S, Wang N, Tan HY, Zhang ZM, Feng YB. The cross-talk between gut microbiota and lungs in common lung diseases. Front Microbiol. 2020;11:301.

69. Zhao F, An R, Wang L, Shan J, Wang X. Specific gut microbiome and serum metabolome changes in lung cancer patients. Front Cell Infect Microbiol. 2021;11:725284.

70. Aguilera-Arreola MG, Ostria-Hernandez ML, Albarran-Fernandez E, et al. Correct identification of Ochrobactrum anthropi from blood culture using 16rRNA sequencing: a first case report in an immunocompromised patient in Mexico. Front Med. $2018 ; 5: 205$.

71. Ryan MP, Pembroke JT. The genus Ochrobactrum as major opportunistic pathogens. Microorganisms. $2020 ; 8(11): 1797$.

72. Comberiati P, Di Cicco M, Paravati F, et al. The role of gut and lung microbiota in susceptibility to tuberculosis. Int J Environ Res Public Health. 2021;18(22):12220.

73. Wu J, Liu W, He L, et al. Sputum microbiota associated with new, recurrent and treatment failure tuberculosis. PLoS One. 2013;8(12):e83445. 


\section{Publish your work in this journal}

The Journal of Inflammation Research is an international, peer-reviewed open-access journal that welcomes laboratory and clinical findings on the molecular basis, cell biology and pharmacology of inflammation including original research, reviews, symposium reports, hypothesis formation and commentaries on: acute/chronic inflammation; mediators of inflammation; cellular processes; molecular mechanisms; pharmacology and novel anti-inflammatory drugs; clinical conditions involving inflammation. The manuscript management system is completely online and includes a very quick and fair peer-review system. Visit http://www.dovepress.com/testimonials.php to read real quotes from published authors.

Submit your manuscript here: https://www.dovepress.com/journal-of-inflammation-research-journal 